COMPTES RENDUS

\title{
PHILOSOPHIE ET SCIENCE
}

Hans-Georg GADAmER, L'Éthique dialectique de Platon. Interprétation phénoménologique du Philèbe. Essai trad. de l'allemand par Florence Vatan et Veronika von Schenck. Le Méjan, Arles, Actes Sud, 1994. 11,5 × 21,5, 324 p. (Le Génie du philosophe).

La dialectique platonicienne est-elle éthique? Telle est la question directrice de la thèse d'habilitation présentée par Hans-Georg Gadamer à l'université de Marbourg en 1928 et publiée en 1931 sous le titre Platos dialektische Ethik (Gesammelte Werke, Tübingen, Mohr, 1991, Bd 5, Teil I). Dans cet ouvrage, l'auteur amorce sa longue réflexion sur ce qui noue l'un à l'autre dialogue socratique et dialectique platonicienne, et désigne le Philèbe comme un lieu privilégié pour l'analyse et l'exercice herméneutiques. Une telle étude s'inscrit dans le débat qui avait lieu alors en Allemagne autour de l'œuvre de Platon sous l'impulsion des travaux de Paul Natorp, J. Stenzel, Werner Jaeger, Paul Friedländler ou encore Samuel Singer. Mais les recherches d'érudition historiques et philologiques demeurent à l'arrière-plan de la lecture entreprise ici qui privilégie le face-à-face direct avec l'œuvre dans le but avoué de sauvegarder l'élément de fraîcheur propre à une investigation immanente et non prévenue. C'est ainsi que la méthode d'interprétation mise en œuvre se veut "phénoménologique » en vertu, d'une part, de l'exemple donné par l'enseignement et les recherches de Heidegger et, d'autre part, de l'injonction husserlienne en faveur d'un accès aux choses mêmes. En fait, si le propos tenu baigne dans les problématiques et le langage de Sein und Zeit, l'inspiration phénoménologique qu'il revendique demeure floue faute d'explicitation méthodologique et doit surtout s'entendre au sens large d'une attention à décrire fidèlement. Cette étude s'enrichit en effet d'analyses suggestives qui scrutent les conditions de l'activité dialogique, puis plusieurs figures concrètes de l'état de plaisir ou de douleur, telles que le manque, la joie, l'espoir, la désillusion ou l'envie.

Le jeune Gadamer revendique ainsi une approche originale de la pensée grecque, libérée de toute scolastique et capable de recueillir dans la langue allemande contemporaine la manière dont les choses se présentaient « en personne » dans cette pensée. La méthode de lecture proposée congédie donc toute focalisation sur la littéralité du texte. En témoigne l'étonnante déclaration introductive, à vocation surtout polémique : "Plus cette interprétation suit de près le texte de Platon, plus elle

Revue de synthèse : $4^{\mathrm{e}}$ S. nos 2-3, avr.-sept. 1998, p. 361-415. 
s'éloigne de son intention de frayer un accès au texte. Et inversement, plus elle prend de distance à l'égard de la terminologie et des orientations platoniciennes, plus elle pense toucher à son but » (p. 18).

L'ouvrage se présente comme un diptyque : une première partie s'attache à dégager la structure de la dialectique platonicienne, une seconde à proposer une exégèse du Philèbe. L'avant-propos de 1931 remarque d'ailieurs que ces deux moments « ne forment pas une unité " tandis que celui de la réédition de 1982 estimera au contraire que l'ouvrage jouit d'une « unité à part entière ». En vérité, selon l'introduction, l'élaboration de l'exposé général sur la dialectique découle de l'examen du Philèbe, "dialogue éthique » qui thématise la pratique dialectique en vue de savoir si, et comment, ce qui paraît bon pour l'homme (le plaisir, le bien-être) l'ouvre au bien lui-même (au Bien en soi). Gadamer ne se cache pas de rapprocher ce texte de l'éthique aristotélicienne et de son attention aux données concrètes de la praxis. Saisie conceptuelle qui s'accomplit en tant qu'effort pour saisir le logos ousias, la dialectique se déploie dans les limites de l'existence humaine, où elle reste précaire et inachevée. C'est alors l'existence humaine elle-même qui apparaît comme possibilité, certes tendue vers un idéal, mais possédant aussi sa forme d'accomplissement propre.

Selon la thèse fondamentale déployée, la dialectique puise son sens dans l'entente dialogique et ménage l'accès à la chose visée. Le logos doit progresser et rendre raison de ses avancées. Ainsi, la parole qui invite l'autre s'identifie à celle qui vise la scientificité. C'est donc la structure de l'entente au sein du dialogue qui sous-tend la théorie de la dialectique. En sorte que, sous l'exigence d'intelligibilité de l'étant, le regard contemplatif se lie à une communication spécifique, voire à un être-ensemble authentique : l'auteur explicite et nuance en ce sens différentes significations du fait de partager la compréhension, de participer en commun à l'enquête discursive, en insistant sur la nécessité de garder en vue l'éclaircissement de la chose, faute de quoi aucune conclusion véritable ne pourra être atteinte. La dialectique définit les conditions d'une entente objective par opposition aux usages pervertis de la parole dont joue notamment l'éristique.

Le Philèbe offre à cette analyse un prolongement concret en même temps qu'il en désigne un lieu d'enracinement. Il montre en effet comment l'aspiration au savoir, directrice pour la question de l'entente, concerne le savoir de la vertu (arêté), qui régit la compréhension qu'a chacun de son existence. La dialectique rejoint donc la façon dont l'existence humaine se justifie. Comment disposer de soi au mieux? L'auteur repère quelques moments où cette question fait irruption dans les dialogues de Platon et y fonde l'impératif d'acquérir un vrai savoir susceptible d'apporter une solution sérieuse à la question du Bien. Réciproquement, le Bien sera principe d'intelligibilité, seul à même de fonder le savoir et de donner sa vraie orientation à l'existence soucieuse de se comprendre. Point nodal identifié par la discussion de la théorie d'Anaxagore dans le Phédon et approfondi par la perspective d'un fondement inconditionné absolu dans la République, la question du fondement demier permet d'exposer l'hypothèse de l'eidos, laquelle vient satisfaire la nécessité de s'assurer de l'unité et de l'identité de l'objet de la recherche. L'Idée du Bien sanctionne ainsi la recherche de l'immuabilité et de la raison dernière des choses à titre de principe ontologique ultime. 
Mais lorsqu'il s'attache à éclairer l'existence morale (son devoir-être et son effectivité), le dialecticien affronte le problème de l'un et du multiple sous la forme du contraste existant entre l'Idée de Bien et les différentes compréhensions de la vertu qui ont cours. À cette fin, son analyse conjugue une " vue synoptique » (synagogê) et une « vue distributrice » (diairésis) selon la célèbre définition du Sophiste $(253 \mathrm{~d}$ ) à laquelle Gadamer rend justice en montrant comment, dans la détermination, unité et pluralité ne s'excluent pas (quoique le problème parménidien de la participation, reconnaît-il, demeure entier). Dans le Philèbe, le travail de division dialectique (distingué d'une dichotomie formelle) avère toute sa fécondité : les unités génériques, principes d'intelligibilité, se décomposent en unités indivisibles qui correspondent aux ultimes différences d'espèce. Dès lors, gage de sa compréhension plénière scientifique et partagée - , la chose est saisie dans son unité et dans sa pluralité. La dialectique rend l'étant concret intelligible en son être. Mais si l'Idée seule détient l'être, le bien de l'existence humaine demeure marqué d'un signe négatif. Face à cette éthique privative, l'auteur montre alors comment la théorie des quatre genres s'oppose à une pure séparation entre l'intelligible et le sensible, et débouche sur la perspective d'un bien non plus inaccessible mais immanent aux choses belles thème plus tard repris en point d'orgue par Vérité et méthode.

Ce commentaire du Philèbe, qui conduira à L'Idée du Bien entre Platon et Aristote (GW 7, III), déjà en rupture affichée avec l'interprétation criticiste de Natorp mais encore sous l'obédience de l'exemple heideggerien (malgré une réticence sensible à réduire le sens ontologique de la pensée grecque à une domination de la présence), fait donc apparaître la précocité de plusieurs des motifs centraux du travail de Gadamer. Ainsi, l'attention de l'interprète à la forme du dialogue l'engage à entrer lui-même en dialogue avec le texte et à révéler, enfouie dans la tradition, une parole toujours vivante. Mais cette réaction déclarée (quoique le nom de Friedrich D. E. Schleiermacher ne soit pas prononcé) à la cécité de lectures trop attachées à l'aspect doctrinal de la pensée platonicienne entraîne l'écrit de 1931 à valoriser l'inachèvement et la nature transitoire de la recherche dialectique (comme mouvement de l'entente) au risque de rendre inintelligibles aussi bien le vœu de vérité qui la dynamise que sa scientificité éminente. En outre, la mise en évidence du socratisme, qui donne à cette valorisation son point d'appui, fait, selon Gadamer, porter le regard vers Aristote et non vers le seul Platon. L'affirmation, plus que la démonstration, de la découverte, dans l'horizon de la question pratique, d'une convergence entre platonisme et aristotélisme, la thèse de leur unité originaire à partir de leur commune filiation avec le « rendre raison » socratique et ses incidences éthiques, conduiront même ce commentaire à s'achever sur l'espoir de servir l'intelligence de l'éthique aristotélicienne. Enfin, la possibilité d'investir l'ontologie à partir de la question du bien pourra paraître une présupposition insuffisamment éclairée. Il est vrai que l'auteur concède à son entreprise de jeunesse le défaut de s'être insuffisamment acquittée de sa tâche, tout en lui reconnaissant le mérite - indéniable d'avoir fourni une impulsion à ses recherches ultérieures.

Un regret : que la traduction proposée, belle voire éclairante (ainsi p. 28 « dogmatique » substitué à « philosophische », Seite 5), ne reprenne pas l'index des noms ni celui des passages étudiés. 
Jacques Brunschwig, Papers in Hellenistic philosophy. Transl. by Janet Lloyd. Cambridge/New York, Cambridge University Press, 1994. 15,5 $\times 23,5$, xiii-277 p., bibliogr., index.

Jacques Brunschwig, Études sur les philosophies hellénistiques. Épicurisme, stoïcisme, scepticisme. Paris, Presses universitaires de France, 1995. $15 \times 21,7$, 364 p., bibliogr., index (Épiméthée).

Depuis quelque temps, la philosophie hellénistique semble se remettre peu à peu du relatif discrédit ayant cours en France et qui cherche à faire d'elle une sorte de parent pauvre de la philosophie antique. Dans ce contexte, la contribution de Jacques Brunschwig mérite incontestablement d'être soulignée et louée, car elle prouve à quel point les thèmes et les textes hellénistiques, loin d'être trop simples, suscitent des discussions philosophiques aussi riches que variées.

La version française et la version anglaise du recueil d'études de Jacques Brunschwig consacrées à la philosophie hellénistique ne sont pas strictement identiques, bien que très proches. Les deux ouvrages rassemblent des travaux publiés auparavant dans divers livres ou revues et sont tous deux divisés en trois parties correspondant aux principales écoles apparues pendant la période : l'épicurisme, le stoïcisme et le scepticisme. Neuf études sont communes aux deux versions: «L'argument d'Épicure sur l'immutabilité du tout », «Épicure et le problème du langage privé », «Remarques sur la théorie stoïcienne du nom propre », « Remarques sur la classification des propositions simples dans les logiques hellénistiques ", "Le modèle conjonctif », « Sur une façon stoïcienne de ne pas être », «Le titre des Indalmoi de Timon : d'Ulysse à Pyrrhon », « Le problème de l'héri-

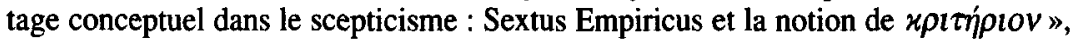

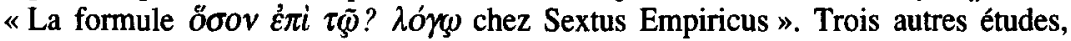
présentes dans la version française, sont absentes de la version anglaise dans laquelle il n'était pas nécessaire qu'elles paraissent puisque publiées à l'origine dans des revues anglaises facilement accessibles au public anglophone. Il s'agit de «L'argument des berceaux chez les Épicuriens et chez les Stoïciens », «Définir la démonstration» et «Sur un titre d'ouvrage de Chrysippe: Que les Anciens ont admis la dialectique aux côtés de la démonstration". Par ailleurs, trois études figurent uniquement dans la version anglaise : "The stoic theory of the supreme genus and Platonic ontology ", "Did Diogenes of Babylon invent the ontological argument?", "Once again on Eusebius on Aristocles on Timon on Pyrrho ». La seule mention des titres des articles laisse entrevoir une diversité certaine dans les thèmes abordés. Néanmoins, il ne s'agit pas d'un «patchwork» philosophique car ces travaux présentent une grande unité dans la manière de traiter les problèmes et de tenter de les résoudre ingénieusement.

Tout d'abord, une caractéristique essentielle de ces recueils est que Brunschwig a toujours le souci de prendre pour point de départ des questions ayant fait l'objet de débats, de discussions et qui soulèvent un certain nombre de problèmes reconnus 
par les commentateurs. Ainsi, le premier article, «L'argument d'Épicure sur l'immutabilité du tout » qui donne le ton de l'ensemble de l'ouvrage en posant certaines exigences de méthode, a pour objet l'étude de cinq lignes de la Lettre à Hérodote d'Épicure qui ont suscité bon nombre de discussions philosophiques chez des commentateurs d'Épicure tels que Jean Bollack, Pierre Boyancé, Carlo Giussani, Ettore Bignone, Cyril Bailey, Olivier Bloch. Chemin faisant, l'auteur est amené à réfuter, infléchir ou confirmer les hypothèses avancées auparavant. Mais dans tous les cas, il importe selon lui de garder toujours à l'esprit la «dette de reconnaissance » qu'il a à l'égard de ses devanciers, dette qu'il souligne dans le premier article : «Sans le travail de nos devanciers, sans l'analyse de leurs efforts, de leurs divergences, de leurs arguments en sens opposés, nous n'aurions assurément pas pu repérer l'existence d'une difficulté [...] ni explorer le labyrinthe des voies sans issue jusqu'au point où l'issue se dévoile et s'impose » (p. 41-42 dans le recueil français). Dans toute la suite du recueil, cette «dette de reconnaissance " est toujours présente: Brunschwig s'efforce de distinguer ce qui peut être retenu et ce qui doit être rejetc des thèses de ses prédécesseurs avec la plus grande rigueur intellectuelle et la plu এ এrande courtoisie. Á titre d'exemple, on peut citer l'article « Le titre des Indalmui de Timon : d'Ulysse à Pyrrhon ". Brunschwig s'interroge sur le sens exact de ce titrc, sachant que le mot Indalmoi est habituellement lié à la notion d'image. Il montre alors comment la solution avancée par un autre commentateur, F. Conrad, mérite d'être exploitée même si cela mène à son dépassement. En effet, il retient l'idée que le titre ne désigne pas nécessairement ce dont on parle dans l'ouvrage mais plutôt la manière dont on en parle. Il s'agirait d'une ceuvre dans laquelle on offre des images plutôt que d'une cuvre dans laquelle on parle d'images. Mais il rejette l'idée que les images désignent des souvenirs (souvenirs que Timon aurait de Pyrrhon), thèse défendue par Conrad qui prend appui sur un passage de l'Odyssée manifestement utilisé par Timon. En se fondant également sur ce texte, l'auteur expose l'idée selon laquelle le terme désigne le décalage qui existe entre Timon et son maître Pyrrhon qu'il représente néanmoins. Les deux personnages sont proches sans être identiques tout comme Ulysse avant son départ et à son retour. Selon lui, c'est en suivant ce type de méthode que l'étude des textes anciens peut encore dévoiler nombre de secrets et de surprises et l'un des intérêts de ce recueil est de le prouver.

Cependant, si l'auteur parvient à ce dévoilement, ce n'est pas uniquement grâce à ses prédécesseurs : c'est aussi, et surtout, grâce au constant mouvement qu'il effectue entre la précision des problèmes abordés et la mise en évidence de la perspective qu'ils ouvrent. En effet, le point de départ de chaque article est toujours très précis : il peut s'agir d'un thème, d'un point de doctrine, de la thèse d'une école ou bien encore d'un ou de plusieurs textes confrontés entre eux, d'une formule précise d'un auteur ou d'un titre d'ouvrage. Le point de départ de chaque article est étudié avec un grand souci de précision et de rigueur logique. Avouons d'ailleurs que le recours à des outils et à un langage logiques rendent parfois certains passages du recueil assez ardus. Ces passages exigent des efforts de la part du lecteur qui se trouve récompensé une fois que s'ouvre l'horizon du problème dans toute l'ampleur que lui donne Brunschwig. Prenons pour preuve l'article "Définir la démonstration ». L'auteur part ici de trois textes précis de Sextus Empiricus dont il démonte patiem- 
ment les rouages pour montrer quelles sont les différentes définitions de la démonstration qu'ils impliquent. Il dégage ainsi de ces textes quatre définitions qu'il distingue avec précision et l'on peut estimer que ces distinctions constituent l'un des passages les plus complexes du recueil français. Puis, dans les dernières pages de l'article, l'auteur, non dépourvu d'humour, change de ton et révèle tout l'enjeu de ce travail minutieux. Il se livre alors à ce qu'il appelle un «petit jeu du portrait-robot» (p. 230-231) : en effet, il dresse le portrait hypothétique de l'auteur de chaque définition. Puis, s'appuyant sur des considérations d'ordre chronologique, il révèle qui se cache selon lui derrière ces portraits. Entrent alors en scène Zénon, Cléanthe et Chrysippe et avec eux, l'histoire du stö̈cisme. À partir de textes précis et d'un problème particulier, c'est donc un aperçu de l'évolution d'un système qui nous est ici offert. On trouve dans d'autres articles d'autres types d'élargissement de la perspective à partir de questions précises. Ainsi, outre l'ouverture sur l'ensemble d'un système considéré dans son évolution, l'auteur tire de points particuliers certaines conséquences concernant l'unité d'un système (par exemple, dans l'article "Remarques sur la théorie stoïcienne du nom propre », il montre l'étroite implication des considérations grammaticales et ontologiques dans le stoïcisme) ou encore

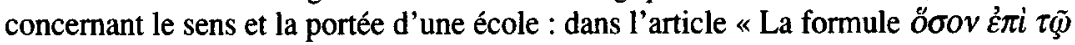
$\lambda o ́ \gamma \omega$ chez Sextus Empiricus », il met cette formule en relation avec le scepticisme dans sa légitimité même et sa possibilité d'exister. Il montre en effet comment le sceptique ne s'interdit pas de parler des phénomènes mais s'interdit d'en parler d'une certaine manière. Il peut arriver aussi que l'examen d'une question débouche sur certaines considérations concernant les relations de différentes écoles : c'est le cas dans l'article « L'argument des berceaux chez les épicuriens et chez les stoïciens ». L'élargissement peut porter aussi sur des aspects d'ordre méthodologique, ainsi que nous l'avons vu concernant le premier article. Enfin, il peut arriver également qu'un texte ou un thème soit replacé dans le cadre de l'histoire de la philosophie, y compris au-delà des limites de la philosophie antique. C'est ce qui se produit dans l'article « Did Diogenes of Babylon invent the ontological argument? », où l'auteur cherche à savoir si l'on peut trouver dans un texte de Diogène de Babylone rapporté par Sextus Empiricus une anticipation du célèbre argument ontologique. La réponse, selon lui négative, est donnée au terme de l'examen des points de vue défendus par d'autres commentateurs, notamment par Jonathan Barnes et Malcolm Schofield. C'est là peut-être un des articles où l'élargissement de la perspective est le plus frappant.

Précisons enfin que seule la lecture de ces deux recueils pourra montrer à quel point Brunschwig parvient à nous convaincre que les écoles philosophiques de la période hellénistique sont loin d'être des écoles assoupies à l'ombre d'un Jardin, d'un Portique ou encore sur « le mol oreiller du doute », pour reprendre l'expression de Montaigne. 
Carlos Lévy, Cicero Academicus. Recherches sur les Académiques et sur la philosophie cicéronienne. Rome, École française de Rome, $1992.17 \times 24,697$ p., bibliogr., index (École française de Rome, 162).

L'objet premier de ce livre, qui reprend une thèse soutenue en Sorbonne en 1988, est de présenter une analyse fouillée des Académiques et de défendre par là même la figure d'un Cicéron philosophe. Mais l'intention qui sous-tend tout l'ouvrage est beaucoup plus vaste : prouver que la Nouvelle Académie ne constitue pas un « intermède sceptique » entre l'Ancienne Académie et Antiochius d'Ascalon (p. 55 et p. 626) et manifester une continuité occultée mais fidèle à l'esprit même du platonisme, à savoir celui d'une raison en travail, fût-ce au détriment de la réaffirmation de thèses fondamentales de l'Ancienne Académie comme l'hypothèse des Formes.

Les enjeux philosophiques d'un tel travail - outre l'évidente mise au point historique des débats entre écoles rivales du $\mathrm{I}^{\text {er }}$ siècle - est de défendre la cohérence de la pensée cicéronienne en dépit de ses détours ou de ses hésitations dialectiques, de focaliser l'attention sur le moteur de l'epoché plus que sur l'epoché même : continuer la recherche, ne pas se satisfaire de positions figées qui témoignent d'une prétention excessive de l'homme dans ses capacités de savoir comme dans la communication qu'il est susceptible d'avoir avec la divinité; bref, défendre un authentique humanisme, modeste mais conscient de ses forces, enraciné dans la nature mais refusant de se diriger vers un naturalisme matérialiste, respectueux de la tradition religieuse sans accepter pour autant la divinisation du sage, et surtout lié de façon très étroite à un enjeu politique qui est la défense de la Rome républicaine : "La condamnation du dogmatisme philosophique sera donc aussi pour Cicéron celle du régime césarien » (p. 633).

La méthode suivie à cet effet combine avec bonheur trois perspectives : l'examen soigneux des textes d'abord et surtout, et la confrontation des Académiques avec les autres traités cicéroniens; la tradition à laquelle se rattache l'Arpinate : le rôle du mos majorum, l'attachement quasi religieux à Platon dont il a traduit deux dialogues, le Protagoras et le Timée; l'expérience personnelle (la mort de sa fille) et la situation historico-politique, la fin de la République et la lutte entre César et Pompée.

L'École philosophique dans l'Antiquité était beaucoup plus qu'un lieu de recherche et d'enseignement : c'était une communauté de vie, « se réclamant d'un maître fondateur dont la présence se perpétuait par l'élection ou la désignation du scolarque » (p. 14), chef dont la liberté d'interprétation pouvait être réduite dès lors que la doctrine de l'École se présentait sous une forme systématique. Or la fluidité de l'enseignement platonicien, la diversité de ses approches et de ses modes d'exposition, donnaient une grande latitude d'innovation au scolarque à condition de rester fidèle à certains principes fondamentaux. D'où le débat interprétatif sur la continuité ou la dérive sceptique des trois périodes de l'Académie : l'ancienne, celle de Platon, la moyenne, celle d'Arcésilas condisciple de Zénon de Cittium (III' siècle), la nouvelle, celle de Caméade, l'adversaire de Chrysippe (11 siècle), et de Philon de Larisse, le dernier scolarque ( ${ }^{\mathrm{es}}$ siècle).

On a souvent présenté la Nouvelle Académie, à laquelle se rattache explicitement et fermement Cicéron, comme une école sceptique, et même comme un dog- 
matisme sceptique, affirmant dogmatiquement que l'homme ne peut rien savoir. La thèse de Carlos Lévy est tout autre : «La Nouvelle Académie est née d'un sursaut, paradoxal dans ses formes mais cohérent dans son propos, des représentants officiels de la tradition platonicienne devant l'apparition de doctrines, le stoïcisme, l'épicurisme, différentes certes, mais ayant en commun de prétendre pouvoir abolir par la sagesse et le bonheur la distance entre l'homme et les dieux [...] Par réaction contre des philosophies de la certitude immédiate, de l'harmonie initiale entre l'homme et la nature, Arcésilas et Carnéade [ont] estimé nécessaire de pratiquer une dialectique qui s'interdisait elle-même toute énonciation positive et ne dévoilait son aspiration à la vérité que par la mise en évidence des contradictions de l'adversaire " (p. 55).

Si l'opposition de Cicéron à l'épicurisme est totale et sans nuances, son rapport au Portique est plus complexe : d'une part, il tend à le réduire à un prolongement du platonisme, d'autre part, il souligne à l'envi les contradictions et l'irréalisme auquel le conduit sa raideur dogmatique et sa façon d'assimiler le sage au dieu; enfin, sur des points particuliers - la théologie naturelle du De natura deorum, la valeur absolue de l'honestum - il adopte comme plus vraisemblables et non, comme le voudrait le système, comme vraies, les thèses du Portique. Lévy montre ainsi (p. 276 sq.) que Cicéron, plus encore que Carnéade, est le véritable inventeur de la position probabiliste, à condition de ne pas donner à ce terme une signification anachronique, en projetant sur le passé nos modèles modernes, statistiques, de la probabilité. Cette position avait certes été préparée par la distinction carnéadienne entre la représentation persuasive (pithanè) et la représentation compréhensive (katalèptikè) et par la préférence accordée par Arcésilas au raisonnable (eulogon) « marque d'une raison consciente de ses limites " sur la certitude et le bien absolus (p. 279-281).

Il ne nous est pas possible d'entrer dans le détail de ces analyses qui constituent une entrée magistrale non seulement dans la philosophie de Cicéron, enfin restituée dans sa finesse, sa complexité et son originalité, mais aussi dans le stoïcisme considéré comme mouvement intellectuel et non pas comme doctrine figée. Nous nous contenterons donc d'indiquer les divers axes de l'ouvrage. Après un examen de l'élaboration des Académiques en ses deux versions successives, de ses structures rhétoriques et de ses sources, l'analyse suit l'ordre des questions philosophiques proposé par Cicéron dans le De divinatione II, afin de mettre en valeur la question la plus essentielle : qu'est-ce que la nature? Il sera donc traité successivement de la théorie de la connaissance, de l'éthique et enfin de la physique qui inclut la théologie naturelle.

L'examen de la théorie de la connaissance est centré sur la question de la représentation : d'où le débat opposant stoïciens et néo-académiciens sur le statut de la représentation compréhensive, la relation entre l'assentiment stoïcien et l'epoché académicienne comme rétention volontaire de l'assentiment, la continuité des notions communes ou prénotions à la sagesse. Ce qui montre le souci de la Nouvelle Académie de se rattacher à la référence socratique en usant de la dialectique non pas pour elle-même mais pour détruire les fausses certitudes et laisser ainsi perpétuellement ouvert le chemin de la recherche.

Le désaccord des moralistes sur la conception du souverain bien suffirait à lui seul à ruiner toute prétention dogmatique à l'accès à une vérité éthique. Mais cette diversité, amplifiée par Cicéron à des fins polémiques, se laisse toutefois ordonner 
par une classification qui distingue entre les fins simples (le bien, le plaisir, l'absence de douleur, la jouissance des biens naturels) et des fins mixtes comme la conjonction de l'honnête et du plaisir. La critique cicéronienne de l'éthique stoïcienne se fonde sur deux reproches : la coupure de l'honestum d'avec la réalité quotidienne qu'il doit pourtant normer et l'excès d'un naturalisme qui enracine la vertu dans les premières tendances de l'être, tout en poussant la divinisation de l'homme jusqu'à l'assimilation du sage à Dieu. Plus réaliste et plus « raisonnable », le platonisme séparait l'ordre éthique de l'animalité de l'homme et limitait l'assimilation à Dieu par un « autant que possible ». En revanche, considérée dans sa dimension collective et politique, l'éthique stoïcienne est réhabilitée puisque Cicéron affirme, en s'appuyant sur des textes du Portique, que le mos majorum des Romains s'identifie à la loi naturelle (p. 517). C'est dans le seul cadre de la cité qu'est possible une certaine « ascension vers la perfection qui caractérise la nature tout entière » (p. 534), seule façon de concilier la transcendance platonicienne avec l'immanence stö̈que.

La « cacophonie des physiciens » se laisse moins facilement réduire que celle des moralistes. L'incertitude des axiomes sur lesquels reposent les démonstrations mathématiques, la dangereuse confusion des hypothèses sur les principes du monde avec des vérités apodictiques, bien loin de la prudence épistémologique du Timée, les difficultés de la théorie de la Providence, les audaces et les incertitudes pernicieuses de la divination, tout cela alimente une dialectique d'apparence sceptique qui exprime comme en négatif, une tendance fondamentale du platonisme : établir sur le mode hypothétique, un modèle d'intelligibilité du tout qui unifie la diversité sensible mais qui ne peut dire l'Être éternel que dans un discours mixte, rationnel et imaginatif. Du Cicéron du De natura deorum qui estime vraisemblable la doctrine du stoïcien Balbus qui se présente elle, comme vérité démontrée, il faut donc dire ce que Lévy dit admirablement d'Arcésilas : qu'il préféra l'absolu de la question à l'absolu de la réponse et s'affirma « sondeur de ténèbres face à ceux qui se prétendaient détenteurs de lumières " (p. 623). Cette dernière citation suffira, nous l'espérons, à donner à tous ceux qui pensent que la philosophie antique $\mathrm{a}$, sinon donné les bonnes réponses, au moins posé d'excellentes questions, pour susciter l'envie de lire un ouvrage qui se signale par son amplitude, sa clarté, son élégance, une connaissance sérieuse de la Quellenforschung qui ne tombe jamais dans l'érudition vaine mais fournit toujours les éclairages nécessaires à l'intelligibilité propre d'une œuvre philosophique souvent ignorée dans sa force et son originalité propres.

Jacqueline LAGRÉE

Joël BIARD, Guillaume d'Ockham. Logique et philosophie. Paris, Presses universitaires de France, 1997. 11,5 × 17,5, 128 p. (Philosophie).

Le $X^{e}{ }^{e}$ siècle voit s'épanouir un mouvement d'idées multiforme, affirmation d'une diversité théorique aussi bien dans le domaine de la logique que dans ceux de la théologie ou de la philosophie naturelle. Guillaume d'Ockham (ca. 1285-1347) prend part à cette «métamorphose du discours scolastique» par la mise en 
cuvre d'une nouvelle façon de philosopher, née d'un rapport inédit entre l'analyse logico-linguistique et les sciences du réel extra-linguistique. Ockham assigne comme tâche propre à la philosophie l'élucidation des discours par l'analyse logique. La triple fonction (propédeutique, critique et productrice) de la logique est ainsi radicalisée, ce qui constitue, avec la place essentielle accordée à la théorie du signe, son apport novateur. Partir de la logique n'est pourtant en rien une position originale pour un philosophe médiéval. Pour Ockham cependant, si l'usage de la logique, entendue au sens large, est bien de discerner le vrai du faux, ce discernement s'exerce sur des propositions : il faut alors nécessairement lier une appréhension formelle des propositions et une appréhension littérale des termes articulés. Ockham philosophe va donc mettre en place une nouvelle architectonique du savoir, qui lie, comme un préambule nécessaire, une théorie du signe à la réflexion sémantique, dans une œuvre systématique de logique. Ockham élabore donc une succincte théorie du signe, sur laquelle il fondera sa sémantique.

La définition ockhamiste du signe semble à première vue reprendre celle, traditionnelle, d'Augustin, en lui ôtant néanmoins la référence au sensible. Cette première modification ouvre la possibilité de concevoir un point de départ intelligible au procès sémiotique. Mais elle introduit surtout la notion déjà constituée de " supposition » : tout signe a une propriété sémantique de suppléance, qui a pour condition une insertion propositionnelle. Par ailleurs, le signe a une dimension gnoséologique : il donne lieu à une connaissance qui peut être une première connaissance, ce que ne permettait pas la définition augustinienne. On ne saurait plus réduire la théorie ockhamiste à une relecture d'Augustin. La connaissance impliquée est la saisie intellective d'un objet, la connaissance même de cette chose. Le concept, conçu comme un acte de l'esprit, est dès lors un signe, pour autant qu'il satisfait à la destination propositionnelle, et le domaine conceptuel peut être soumis à l'analyse logico-linguistique. Si Ockham pose trois sortes de signes linguistiques, et donc trois sortes de langages, c'est néanmoins le terme mental qui retient le plus son attention. Signe naturel et non conventionnel, il fonde sa référence à son signifié dans une relation de causalité. Là encore, si le terme similitudo est employé pour désigner le concept, le modèle augustinien de la «similitude » entre le signe et la chose tend pourtant à s'effacer au profit de celui de la causalité.

Cette évolution se fait conjointement à celle qui affecte la théorie de la nature du concept : Ockham mène en effet une polémique de plus en plus explicite contre la notion de fictum, être objectif mais non réellement distinct, qui serait par exemple celui du concept. Le concept étant de plus en plus nettement pensé comme un acte signifiant, la thématique d'une "ressemblance » désormais inconcevable entre un acte et une chose disparaît naturellement. La « similitude » maintenue entre l'intention et la chose doit être distinguée de la « ressemblance", qui joue par ailleurs un rôle dans la formation du concept commun. À l'encontre également de toute la tradition aristotélicienne, la relation de signification directe ne s'établit pas pour Guillaume d'Ockham entre les mots et les intentions. Au prix d'une réinterprétation du Philosophe, Ockham soutient que le concept signifie d'abord la chose elle-même. C'est donc dans une relation immédiate que tout signe linguistique, donc aussi le signe mental, suppose pour son signifié. Or ces signes se définissent par leur propriété à suppléer quelque chose dans des propositions : il faut donc faire la théorie de l'usage de ces termes, c'est-à-dire des modes de signification et de référence. 
L'analyse des propriétés référentielles des termes d'un langage s'appliquera de la même manière aux trois langages. La Somme de logique a en effet constitué le domaine conceptuel en un langage mental, premier par rapport au langage parlé, mais comme lui formé d'unités morphologiques articulées sur un double axe : référentiel, d'une part, et syntagmatique, d'autre part. Construit par idéalisation du langage parlé, le langage mental, dont les éléments sont des notions de portée universelle, doit pouvoir rendre compte de tous les discours producteurs de savoir. Élaborer une sémantique revient à analyser deux relations entre mots et choses singulières : la supposition et la signification. Ockham traitera donc de modes de significations là où une illusion réalisante a conduit les métaphysiciens à parler de modes d'être. Ainsi est-ce le signe, non le signifié, que l'on peut dire universel. On voit ici comment le bon usage de la logique détruit les illusions nées d'une métaphysique qui assigne à chaque nom distinct un signifié distinct propre. La fonction critique de la logique exerce ici son effet destructeur, reléguant au rang de faux problème la «question des universaux ». De même, les catégories n'ont plus qu'une portée sémiologique, exception faite des catégories de substance et de qualité qui ont une portée absolue (elles supposent pour des choses absolues). Comme l'universel, elles sont des signes " de seconde intention " qui permettent de classer les signes de première intention, mais en aucun cas les êtres. La dissipation des illusions métaphysiques s'accompagne d'une élaboration métaphysique positive, la logique exerçant de manière simultanée une fonction critique et une fonction créatrice. Une ontologie du singulier, fondée sur la thèse de la singularité de tout étant, se met en place, qui échappe aux abus dévoilés par l'analyse de la signification. La théorie des modes de supposition est une théorie de l'usage des termes, elle donnera donc lieu à une division des modes de supposition. La supposition prime donc à première vue sur la signification, qui est une «supposition potentielle». Cependant, celle-ci est préalable à toute étude concrète de la supposition d'un terme. Leurs variations sont donc corrélatives et dépendent du contexte.

Cette théorie du langage mental est le fondement logique, dans l'architectonique du savoir, de la théorie analytique du jugement (celui-ci portant sur des propositions se rapportant aux choses selon différents modes) développée dans les Commentaires des sentences, et de la théorie de la formation du concept, signe mental. L'analyse du jugement et la synthèse décrivant le procès de formation du concept forment les deux pans d'une théorie ockhamiste de la connaissance. Selon la définition d'Ockham, le jugement est un acte par lequel l'intellect accorde ou refuse son assentiment à une proposition mentale. Il se distingue donc de l'appréhension, dont il peut être contemporain, mais qu'il présuppose. Or cette notitia, saisie par l'esprit, est dite évidente quand elle connaît une vérité complexe «par la simple connaissance des termes ". Nous sommes donc en présence d'une raison fondatrice médiate, qui est appréhension de termes et non d'êtres singuliers. La construction de l'édifice du savoir appelle donc la théorie du rapport de ces termes aux choses, à partir duquel il devient possible de classer les différents types de connaissances. Par ailleurs, si l'acte de juger est purement intellectif, il n'en demeure pas moins que le savoir humain est conditionné originairement par une « cause partielle » : la saisie sensible du singulier; la seconde cause partielle étant l'intellect lui-même. L'enchaînement de présuppositions (la relation à la chose signifiée conditionne l'appréhension du terme simple, qui conditionne l'appréhension d'un complexe et le jugement) ne pro- 
duit donc qu'une connaissance propre et directe du singulier. Le lien originel avec le sensible n'est pas un ingrédient mais une condition de la connaissance, qui est une production intellective pure; de même qu'une saisie sensible peut être pure de saisie intellective. Ces deux connaissances se terminent à la même chose : l'être singulier en son essence.

Cependant, nous pouvons appréhender des termes ou des propositions mentales : ces deux modes de saisie des termes vont être appelés « connaissance intuitive » et " connaissance abstractive ". Là encore, il s'agit de deux actes gnoséologiques distincts par leur nature et non par leur objet. Ockham soutient de façon polémique qu'il n'y a pas d'objet propre à l'intellect qui serait un intermédiaire inutile dans la formation du savoir : le sujet du savoir est toujours un être singulier saisissable sensiblement. Pourtant, il y a bien un passage à une connaissance générale, complexe, indifférente à la présence de la chose, dont il faut rendre compte. Par une réaction psychophysiologique de l'intellect, la connaissance intuitive est immédiatement redoublée par une connaissance abstractive commune, « confuse » en ce qu'elle est indifférente à toute singularité, tout en restant connaissance d'une singularité. Cette première connaissance abstractive engendre un habitus, grâce auquel la réitération de la connaissance abstractive est possible sans l'accompagnement de la connaissance intuitive. La caractéristique principale de la connaissance abstractive est donc son abstraction à l'égard de l'existence de son sujet, par conséquent à toute détermination spatio-temporelle. Par là, elle est générale. L'intellect lui-même possède néanmoins une dimension spatio-temporelle par l'intermédiaire de la mémoire. Il s'agit toujours de la remémoration d'un acte intellectuel par lequel je jugeais de l'existence ou non d'une chose. La temporalité est donc présente dans l'âme intellective, indépendamment de la présence effective de la chose.

À l'intérieur du domaine de la notitia, le vocabulaire de la cognitio scientifica délimite un domaine plus restreint. La scientia est la « connaissance évidente d'une vérité nécessaire", «à même d'être produite par une autre connaissance complexe ", une disposition de l'esprit. Or l'apodicticité du savoir pose problème dans un univers chrétien marqué par la contingence radicale des créatures. Nous n'avons en effet de science que de conclusions démontrées à partir de prémisses dont on a soit une science (la prémisse est elle-même au terme d'une argumentation rationnelle), soit une saisie intellective. Pour Ockham ce sont bien les conclusions qui sont nécessaires, indifféremment à la contingence du sujet de la proposition : la nécessité du savoir est déplacée au niveau logique. Certes, toute proposition renvoyant à des choses contingentes est contingente et ne peut avoir le statut de conclusion démontrée; elle peut néanmoins faire partie d'une démonstration. Objet d'une connaissance évidente, on peut former au sujet des choses pour lesquelles elle suppose certains types de propositions nécessaires. Tout discours peut être producteur de science s'il appelle l'assentiment requis. Inversement, seuls les énoncés donnant lieu à cette disposition de l'esprit seront sus au sens strict, quelle que soit la discipline constituée qui les propose. La science porte donc sur des propositions dont les éléments supposent pour des choses : l'analyse logico-linguistique va naturellement s'éprouver au contact de la théologie, dont un certain nombre de propositions ont Dieu pour sujet, et de la philosophie naturelle, dont certains sujets (la matière, le mouvement, la causalité) offrent des difficultés d'appréhension. 
La question du discours sur Dieu est posée par le biais du problème des noms de Dieu, pensés comme ses attributs. Les attributs sont des intentions de l'âme et renvoient donc à des choses absolues réellement distinctes du sujet. Par conséquent, tous les concepts attribués à Dieu sont communs à Dieu et à la créature, et seul un concept complexe formé à partir de nombreux concepts abstraits peut être le concept propre de Dieu. N'ayant pas de saisie intellective de Dieu, nous ne pouvons connaître son existence, ni en faire le sujet d'aucune proposition évidente. Des démonstrations partielles peuvent être envisagées, mais aucun attribut de Dieu, non plus qu'aucune de ses propriétés ou aucun article de foi ne peuvent être démontrés. La théologie n'est pas constituée comme science. L'application à la théologie de l'analyse logico-linguistique délimite les domaines de la science et de la foi, ménageant trois espaces : ceux des croyances pures, des arguments persuasifs et de la science. La mise à l'épreuve de la philosophie naturelle conduit de son côté à une modification du statut théorique des principales catégories de la physique. La notion de substance n'est plus qu'une manière de se référer à quelque chose, tandis que la notion de quantité est elle aussi déréalisée. Outre la mise en cuvre systématique d'une critique du langage (qui conduit, par exemple, à récuser la réalité du mouvement), la critique de la philosophie naturelle fait apparaître les limites des domaines du savoir et de la connaissance. Ainsi l'absence de connaissance propre de la matière n'empêche-t-elle pas que nous puissions établir son existence par le raisonnement.

En conclusion, le nouveau rapport de l'analyse logico-linguistique aux sciences du réel, rapport systématique, donne naissance à une critique toute-puissante, confiante. Celle-ci dissout une métaphysique, pour mieux en fonder une nouvelle. Elle est une critique de la raison linguistique, critique du langage fondatrice qui commande toute réflexion humaine. Cette raison philosophique, autonome, puissante à détruire les illusions, est le seul étalon de vérité et d'une stricte scientificité. La philosophie occupe désormais le cœur de l'édifice du savoir. Guillaume d'Ockham rompt définitivement avec la théologie du verbe augustinienne, tout en s'inscrivant fermement dans un mouvement de pensée général qui ébranle les fondements du modèle cosmologique aristotélicien. Le nom qui se voulait moqueur de Venerabilis Inceptor lui sera donné à juste titre, lui qui, armé de la confiance du débutant, fut l'initiateur d'une nouvelle manière de philosopher.

Sophie AUDIDIÈRE

Paul JACOPIN et Jacqueline LAGRÉE, Érasme, humanisme et langage. Paris, Presses universitaires de France, 1996. 11,5 × 17,5, 128 p., bibliogr. (Philosophies).

Cet ouvrage s'attache à la question du langage dans la pensée d'Érasme, sous différentes perspectives, qui se veulent néanmoins chacune une approche de la question de l'humanisme. On notera donc, dès l'abord, une mise au point utile sur ce thème controversé et un traitement efficace de la spécificité de l'humanisme érasmien, envisagé par le biais du langage.

Les auteurs le soulignent, en effet, dans l'introduction comme dans la conclusion : leur projet s'est voulu «modeste» (p. 4), le point de vue est « volontairement 
restreint et focalisé sur la question du langage », il ne s'agit en aucun cas de " porter un jugement d'ensemble sur la pensée d'Érasme » (p. 105). Cependant, le langage apparaît à la lecture de leur ouvrage comme un moyen d'insertion heureux dans la pensée philosophique et chrétienne d'Érasme.

L'exposition procède en trois temps. Tout d'abord, il s'agit de mettre au jour le rôle fondamental (au sens strict : il s'agit du fondement) du langage dans la formation de l'homme (accroissement des connaissances, mais aussi et surtout accès à la vertu et à la liberté), en insistant sur le choix érasmien des langues et des lettres antiques, notamment latines. Puis, le lien de la formation en général et de la formation chrétienne chez Érasme nous amène au rapport du langage et de la religion : la théologie authentique exige un rapport direct aux textes sacrés (d'où l'œuvre érasmienne majeure de lecture, de traduction et de commentaire), qui ne peut être bénéfique que par la maîtrise des langues anciennes, laquelle, soulignent les auteurs, ne peut être le fait que d'une élite, et non de la foule. D'où, dans un troisième temps, à partir de ce partage entre la foule et l'élite, le traitement de la question de la relation entre la langue et la société, moment où éclate la contradiction majeure sous-jacente à la pensée érasmienne. Non seulement le langage est la condition de la pensée (dans l'éducation et la religion), mais il est également la condition de la vie sociale. Le mensonge par exemple (dont relève le souci de gloire) détruit celle-ci, alors que le postulat du droit à la parole ou l'art de la conversation la servent. Cependant, le choix érasmien du latin est mis en exergue comme porteur d'ambiguités - le souci de la société semble aller contre la constitution nécessaire d'une élite linguistique, quant à la volonté de faire du latin une langue vivante, elle semble contradictoire en elle-même, au moment de l'essor des langues vernaculaires - et de fermetures (fermeture de la société des lettrés sur elle-même, fermeture de l'influence de la pensée érasmienne).

Finalement, à partir d'une conception « simple », voire « simpliste » de la langue par Érasme (p. 110), les auteurs ouvrent de nombreuses perspectives sur la philosophie érasmienne, multiplient les angles d'insertion dans sa pensée, dans un ouvrage clair et synthétique.

Sophie Peytavin

Frédéric Brahami, Le Scepticisme de Montaigne. Paris, Presses universitaires de France, 1997. 11,5 × 17,5, 126 p., bibliogr. (Philosophies, 83).

Le renouveau des études sur Montaigne est dû en bonne partie à la réappropriation des Essais par les philosophes. En guise d'exemple, la collection "Philosophies ", dont fait partie le livre de Frédéric Brahami, publie, à moins de quelques mois d'intervalles, deux essais sur la pensée de Montaigne. Un premier titre, celui de Ian Maclean, s'attachait à commenter la rhétorique philosophique de Montaigne, en la comparant avec les autres degrés de discours à l'œuvre dans les Essais. Brahami s'intéresse plus précisément à l'« Apologie de Raymond Sebond », qu'il place en droite ligne avec la tradition issue du scepticisme ancien. Puisque toute la ques- 
tion du scepticisme est de comprendre les modes de la croyance, il est naturel que ce problème débouche chez Montaigne sur celui des rapports entre foi et raison. On voit ainsi comment le discours de Montaigne, du projet de défendre Sebond, se trouve en position de condamner une œuvre qu'il considère dogmatique. Il ne s'agit pas non plus de nier les effets de la grâce. Le scepticisme de Montaigne est ainsi une réaction contre les facilités du dogmatisme et une redéfinition de la croyance et du jugement.

Le scepticisme est une pensée des conditions de l'affirmation. Toute la question de la vérité des énoncés du discours est contenue dans ce problème. Si le langage ne peut mener l'homme qu'à l'erreur, on peut penser que la solution la plus juste est la résignation dans l'absence du jugement, l'epoché grecque. Mais il faudrait alors admettre un échec du langage, alors que c'est par lui que l'âme s'ouvre à la présence divine. C'est que la raison du langage n'est pas le tout du langage : les apories de la rationalité ne sont pas les reflets d'un échec de l'homme mais au contraire l'épanouissement de son être dans sa plénitude, c'est-à-dire dans les divers mouvements qui traversent sa vie qui seront eux aussi exprimés dans le langage. La pensée tire ainsi son existence paradoxale d'une négation de sa suprématie, ce qui permet à Brahami d'affirmer qu'ainsi Montaigne fait place libre à une nouvelle théologie, d'où, étrangement, le dogmatisme est banni et ce, sans perte pour le dogme. Comment cette négation ne conduit-elle pas à la destruction de la pensée mais au contraire favorise-t-elle son activité? La raison étant incapable de se figer dans la certitude, elle ne peut jamais être passive et ne faire que recevoir ou refuser les objets qui lui sont proposés. Brahami explique avec rigueur comment la dynamique de la raison s'explique par la capacité propre à l'esprit de ne pas exclure une représentation d'un objet quelconque au moment où intervient une autre représentation du même objet, mais de tirer profit de leur rencontre. Faut-il alors comprendre que le scepticisme soit incapable d'une pensée pratique? Dans une section du livre consacrée à la critique du pyrrhonisme par Montaigne, Brahami commente la « substitution de l'asthénie à l'isosthénie ». Dans le premier cas, celui de l'asthénie, la valeur des jugements sur les objets ne peut être mesurée puisque les jugements ne sont jamais comparés entre eux mais simplement mis en parallèle les uns avec les autres. L'isosthénie, au contraire, en attribuant aux jugements un degré de valeur qu'elle ne peut en aucun cas justifier, oblige à une reprise constante de l'acte de juger. En supposant d'un jugement une valeur de vérité supérieure à celle attribuée à un autre jugement, le sujet se voit obligé de reprendre sans cesse ses investigations puisque les raisons de ses choix sont sans cesse démenties. À la dynamique de la reprise éternelle du même, Montaigne préfère l'inventaire des possibles. Ici, le choix ne se fait plus et la raison ne fait que permettre au sujet d'emmagasiner les représentations. Dès lors, ce n'est plus seulement l'objet qui subit l'examen de la critique, c'est la faculté même de juger. La multiplication des hypothèses vide de sens la prétention au savoir définitif. Comme l'explique Brahami, la disparition de l'isosthénie implique aussi celle de l'epoché, la suspension du jugement, car l'âme se transforme elle-même au rythme de la dérive des objets qu'elle regarde. L'âme n'est pas dissociée des objets qu'elle se représente et subit donc leur transformation. En somme, le scepticisme est moins chez Montaigne une critique de la rationalité qu'une affirmation de la vie, c'est-à-dire le constat d'une dynamique des oppositions présente en 
toutes choses. Loin de rechercher l'équilibre tranquille de son âme, l'homme de Montaigne est sans cesse secoué par ces oppositions et vit par celles-ci.

Le scepticisme grec est un rationalisme où la recherche de la vérité s'effectue dans les capacités réflexives de l'âme. La disposition par l'esprit des objets qui lui sont offerts n'est faite qu'en fonction d'une introspection de l'âme. Brahami explique comment le christianisme empêche la circularité de l'esprit et oblige à rechercher la vérité au-dehors de l'esprit humain, puisque la vérité est dans $\mathrm{Dieu}$. $\mathrm{Si}$ l'âme n'offre pas l'intelligence des choses mais nous informe plutôt sur notre rapport moral aux objets, ce n'est plus la réalité de ceux-ci qu'il faut interroger mais les raisons qui motivent le désir de les connaître. L'anthropologie chrétienne ne place plus alors au centre de son système la raison mais la volonté et le désir. C'est la volonté qui dirige l'intelligence et non l'inverse, d'où l'impossibilité de la contemplation intérieure. Ce qui est à contempler est hors de soi. Mais de même que l'intelligence ne peut prétendre contraindre l'âme à contrôler ses passions, de même elle ne peut contrôler ce qui lui est extérieur, ce qui rend le don de la foi absolument nécessaire. Comme la raison ne peut pas être le guide de la croyance, c'est bien la vie, au sens où nous l'entendions plus haut, qui servira de fondement à une nouvelle compréhension de la foi.

Au sujet de la foi de Montaigne, Brahami s'écarte des études qui tendaient à rapprocher Montaigne de l'augustinisme baroque. Lecteur de saint Augustin, Montaigne ne s'en sépare pas moins sur plus d'un plan. Montaigne refuse l'idée selon laquelle Dieu ne peut être en contradiction avec lui-même : ce serait affirmer que Dieu est prisonnier d'une nature qui lui est assignée par l'homme. Le scepticisme de Montaigne s'affirme bien ici contre le dogme religieux, et non contre le dogme de la foi : ce n'est pas la nécessité de la croyance qui est attaquée, mais la prétendue rationalisation de cette nécessité. La pertinence même de la théologie, qui suppose un dialogue permanent entre l'intelligence divine et l'entendement humain, est remise en cause. Le Dieu de Montaigne est au-dessus des contradictions supposées par les théologiens être inhérentes à la nature divine, puisque ces dernières ne sont toujours le fait que du seul entendement humain. Ici, le scepticisme devient fidéisme, un déni radical du rationalisme. Il ne s'agit plus de douter de l'existence de la vérité ou d'en reprendre sans cesse la recherche, mais de la situer dans un ailleurs inaccessible pour l'homme, ce qui revient à cesser de vouloir comprendre Dieu pour ne faire que croire en lui. Mais cette impossibilité n'a pas seulement des conséquences sur la foi : le fidéisme de Montaigne participe pleinement à son anthropologie en ce sens que la possibilité même de connaître la vérité ou l'essence des choses est retirée à l'homme, cette vérité n'appartenant toujours, en dernière instance, qu'à Dieu seul. Ainsi, toute l'intelligence du monde ne peut-elle reposer que sur l'illusion de l'existence des objets. Mais à l'encontre de la doxa, la foi n'est pas contigente, puisque son objet existe au-dehors de sa compréhension par le sujet. Que les modalités de la croyance soient le fait de l'irrationalité des hommes, Montaigne l'admet sans peine, puisque la seule véritable raison n'est pas celle de l'homme mais celle de Dieu. Quel rôle alors peut-on accorder à la raison? Contre Sebond, Montaigne refuse le primat de la raison non seulement pour l'intelligence du divin mais pour celle de tout objet. Avec Sebond, Montaigne soutient que la raison peut servir la foi, non pas de manière protreptique, comme le pensait Sebond, mais en tant que lumière per- 
mettant de mieux vivre avec la Révélation. La raison tout au plus poursuit mais ne précède jamais l'œuvre de la croyance.

On regrettera toutefois une exagération dans la singularisation de la figure sceptique de Montaigne, au sujet de sa critique radicale du jugement notamment, qui rend suspecte l'association obligée - au moins depuis les travaux classiques de Popkin - de sa pensée et de la philosophie sceptique des $x v I^{e}$ et XvIII siècles. Il est certes impossible d'imaginer un même fidéisme chez Descartes ou chez Hume. Brahami suppose qu'il fallait dépasser le scepticisme ancien, dont l'articulation théorique ne menait nulle part. Ce n'était pas la faillite des jugements, trop liés aux circonstances, que remettait en cause Montaigne, mais l'acte même de juger. Chez Montaigne, l'esprit n'est pas au-dehors de l'expérience mais participe à l'activité de celle-ci et se soumet aux mêmes conditions qu'elle. On peut supposer que c'est à partir du principe de vie que l'anthropologie de Montaigne peut être reliée à la science expérimentale de l'Âge classique et plus encore, de l'époque des Lumières.

Christian Nadeau

Olivier Mllet, La Première Réception des Essais de Montaigne (1580-1640). Paris, Honoré Champion, 1995. 15,3 × 22,5, 252 p., bibliogr., index (Études montaignistes, 23).

Cette publication présente un double intérêt. Tout d'abord, s'inscrivant en faux contre l'idée, généralement admise, que les Essais de Montaigne n'ont pas rencontré l'accueil qu'ils appelaient eux-mêmes auprès du public lettré du premier xvil siècle, Olivier Millet s'attache, dans un court mais dense essai introductif, à reconstituer la généalogie de la réception critique du "Sénèque françois ", depuis la première édition, en 1580, jusqu'à la génération de Gabriel Naudé et de Blaise Pascal, en passant par les années 1620, quand le magistrat-écrivain fut associé à Pierre Charron, ce qui compromit sa réputation dans le cadre de la polémique contre les « libertins érudits ». Preuves à l'appui, $\mathrm{O}$. Millet nous montre que, durant la période qui conduit des dernières manifestations de l'humanisme de la Renaissance au seuil de l'Âge classique, les Essais ont fécondé une part de la création et de la critique littéraires, participant ainsi à la " naissance de l'écrivain ${ }^{1}$ ". Le second intérêt de cette publication réside dans l'abondant dossier documentaire, qui constitue l'essentiel du livre (p. 49-236) : voici, en effet, systématiquement rassemblés, des textes jusque-là peu accessibles ou connus de façon fragmentaire. Plusieurs sont pour la première fois traduits ou reproduits, comme la longue « Lettre à Achante » de Jean-Pierre Camus. De Pierre de Brach, en 1576, à Gabriel Naudé, avec ses Considérations sur les coups d'État (1639), l'ordre chronologique choisi nous fait assister à la naissance d'une tradition critique et de ses variations.

1. Alain VIALA, «Les Institutions de la vie littéraire au XVII ${ }^{e}$ siècle ", dactylogr., thèse de l'université de Lille, 1985; ID., Naissance de l'écrivain. Sociologie de la littérature à l'Âge classique, Paris, Minuit (Le Sens commun), 1985. 
Cette étude apporte ainsi une contribution importante au problème de la réception d'une cuvre, à l'histoire et aux formes du jugement critique dans le domaine littéraire. On lira, à ce sujet, les pages consacrées à la comparaison de quatre lectures des Essais (p. 18-22). Madame de Gournay, tout d'abord, « fille d'alliance de Montaigne ", suivant ses propres termes, préfacière de plusieurs éditions, de 1595 à 1635 , se présente comme une autodidacte revendiquant sa féminité : «C'est une femme qui parle. » Et elle salue dans l'œuvre de Montaigne un accomplissement moderne des valeurs humanistes dont elle se dit héritière. Étienne Pasquier, quant à lui, est le prototype du magistrat érudit : aussi se montre-t-il sévère sur le mépris des convenances, en particulier linguistiques et morales, dont fait preuve Montaigne. Mais il le « sauve », au nom d'une solidarité de magistrat et de la philosophie stoïcienne, largement partagée dans le milieu de la robe ${ }^{2}$. Disciple de Juste Lipse, Dominique Baudier est un humaniste international : il s'exprime en latin, en fonction d'un «canon » critique représenté, entre autre, par Quintilien et Sénèque. Comme l'explique $\mathrm{O}$. Millet, l'éloge de Montaigne devient possible dans ce cadre culturel, mais au prix d'une ignorance complète du projet personnel et nouveau de la peinture du moi. Jean-Pierre Camus est le dernier représentant de ce quatuor de lecteurs érudits et critiques à l'aube du Grand Siècle ${ }^{3}$. Exemple de prélat prédicateur, chargé d'enseigner et d'édifier, ce qui l'intéresse dans Montaigne, c'est avant tout la «peinture du moi » - il est le seul en son temps à l'approuver —, l'homme privé soucieux de " réformation » intérieure : «C'est un autheur autant sçavant en l'art de vivre qui s'en puisse voir et qui s'est descrit autant religieusement que franchement que fit jamais homme; nul, à mon advis, l'a égalé en ce poinct, non que surpassé. »

On le voit, ces quatre lectures différenciées de Montaigne obéissent, chacune, au déterminisme d'une position sociale et d'un milieu culturel qui expliquent la nature du jugement, la position critique adoptée, mais aussi les occultations et les choix de lecture d'un texte qui continue à vivre dans le regard qui lui est porté.

Joël CoRnETte

Geneviève Rodis-LewIs, Descartes. Biographie. Paris, Calmann-Lévy, 1995. 15 ×23, 371 p., index.

Il y a quelques années, j'avais été conduit à présenter dans la Revue philosophique $(3,1985$, p. 320-325) un compte rendu du Descartes de Geneviève RodisLewis paru en 1984 (Librairie générale française, coll. " Le Livre de poche "). Mon compte rendu passait sous silence, par souci de brièveté, toute la première partie de cet ouvrage d'ensemble, consacrée à « Vie et développement de l'œuvre », sorte de condensé de ce qui devra plus tard constituer une biographie très détaillée, dans le même esprit.

2. Gerhard Oestreich, Neostoicism in early modern State, trad. de l'allemand, Cambridge, Cambridge University Press, 1982.

3. Sur Jean-Pierre Camus, voir l'édition des Homélies des États-Généraux (1614-1615), texte établi et commenté par Jean Descrains, Paris/Genève, Minard/Droz, 1970. 
Un seul élément matériel nouveau, « découverte très récente ", dans la biographie (p. 71), renvoyant en note au "Bulletin cartésien » des Archives de philosophie, année 1991 : il s'agit d'un ouvrage de Pierre Charron aimablement dédicacé par un ancien, le père Molitor, S.J., " au très savant, cher ami et petit frère Descartes (Cartesio) ». Peut-être le Jésuite a-t-il voulu offrir au jeune Descartes, en cette fin de 1619 , comme une sorte de contrepoison à son ambition de savoir total. Précisément, à ce moment, le philosophe se trouvait, selon l'Abrégé de l'abbé Adrien Baillet (1692), à Neuburg - sur le Danube, au nord de la Bavière, et non pas à Ulm comme on l'a cru (cf. la note 108 dans G. Rodis-Lewis, L'Euvre de Descartes, Paris, Vrin, 1971, p. 448).

Quelques remarques sur les sources. La principale est l'abbé Baillet, auteur d'une Vie de M. Descartes en 2 vol. (1691) ainsi que d'un Abrégé sur le même sujet (1692). Généralement bien documenté, citant ses prédécesseurs et le plus souvent ses références, non sans parfois des confusions, notamment de dates, qui, inaperçues, induisent par voie de conséquence «l'invention », dit G. Rodis-Lewis (Descartes, "Livre de poche », p. 35), d'un séjour parisien inexistant de 1612 à 1618, d'où la nécessité de lire avec d'autant plus de vigilance et d'esprit critique l'indispensable abbé Baillet. En ce qui concerne les éditions modernes du philosophe, la Biographie (p. 9) note la grande édition Adam-Tannery, enrichie dans une réédition récente par les soins du regretté Pierre Costabel (Paris, Vrin/CNRS, 1964-1974), mais G. Rodis-Lewis ne cite pas l'éditeur de « La Pléiade » (André Bridoux) dont la deuxième édition reste très partielle, surtout pour les textes qui suivent et illustrent le Discours, ainsi que la si importante correspondance. La totalité des textes de Descartes devait, vers fin 1996, commencer à être publiée (au besoin en traduction française) par Jean-Marie Beyssade dans la « Bibliothèque de la Pléiade » (ce qui, à ma connaissance, n'a pas eu lieu). Peut-être le travail, considérable pour les huit volumes prévus, est-il aujourd'hui encore en chantier.

René Descartes est né le 31 mars 1596 à La Haye en Touraine, fils de Joachim Descartes qui avait acheté en 1585 une charge de conseiller au parlement de Bretagne et de Jeanne Brochard, son épouse, de santé fragile, qui devait décéder dès 1597. Joachim se remaria vers 1600 . On fit croire à René, avec cette sorte d'indifférence, courante à l'époque, pour l'état d'enfance, d'abord qu'il était né avant terme, ensuite qu'il avait coûté la vie à sa mère, en lui laissant ignorer l'existence d'un frère puîné qui survécut très peu et dont René n'entendit jamais parler.

Ses études au collège de La Flèche (créé en 1604, mais sa faible santé lui fit octroyer un délai après une première éducation due à la grand-mère maternelle et un statut privilégié, une chambre particulière et un repos quotidien surtout) eurent lieu de Pâques 1607 à septembre 1615. Il passa le baccalauréat et la licence en droit à Poitiers, en octobre 1616; dates et séjour inconnus de Baillet. Après s'être émancipé d'éventuelles pressions familiales, il séjourna à Breda (Pays-Bas) de début 1618 à fin avril 1619; il choisit durant cette période le métier des armes, en s'engageant dans les troupes de Maurice de Nassau; il ne tira de cet engagement quasiment aucun avantage et comme il détestait déjà, semble-t-il, les aventures guerrières en même temps que les débauches de la soldatesque, il reprit sa liberté « grâce aux rentes et propriétés héritées de sa famille » (p. 39). Il aimait pourtant les armes et les avait déjà pratiquées au collège où sa santé s'améliorait, stimulée par une vivacité naturelle de tempérament. 
On passera sous silence l'extrême détail du récit biographique tant à l'égard des incertitudes qui demeurent que pour ce qui est des corrections apportées avec sûreté aux innombrables légendes dont on tisse ordinairement la vie de Descartes. Ce lourd volume exige de qui s'y intéresse une lecture directe.

C'est à Breda qu'eut lieu la première des multiples rencontres avec Isaac Beeckman (datée dans le Journal de ce dernier du 10 novembre 1618). Les deux hommes s'admirèrent réciproquement, l'un étonné par ce jeune cavalier si précocement formé aux mathématiques, l'autre convaincu par l'exemple de son aîné qu'une science nouvelle était possible par l'étroite union physico-mathématique des intuitions rationnelles, loin de toute mécanique formelle. Baillet a raconté cette première rencontre (cité dans le Descartes du «Livre de poche», p. 42-43). Cette ardente amitié dura jusqu'en 1630, moment où Descartes expédia à son ancien ami une violente et vraiment féroce lettre de rupture (aucune allusion à l'apport providentiel de Beeckman ne subsistera dans le Discours de 1637).

Une autre rencontre, non moins capitale sur le plan des fondements métaphysiques de la certitude rationnelle fut, à l'encontre des craintes d'impiété dans le cercle de Mersenne, celle du principal artisan de la réforme catholique, le cardinal de Bérulle, nonce du pape à Paris, qui, probablement pendant l'hiver 1627-1628, se montra sensible au talent du jeune mathématicien et l'encouragea, sans doute de façon décisive, à se délivrer de ses doutes sur la légitimité de sa recherche philosophique.

Auparavant il avait beaucoup voyagé, tant en France qu'en Allemagne, par curiosité (aussi en Italie, par dévotion à la Vierge, à l'été 1620) et en se tournant progressivement vers sa propre vie intérieure comme source et fondement primordial de toute vérité, y compris d'expérience. À part quelques voyages en France (étés 1644, 1647 et 1648), il demeura en Hollande à partir de 1628, pour sauvegarder la liberté solitaire de sa réflexion. Il publia, en 1641, son œuvre principale, les Méditations, avant d'accepter, par déférence et en fait pour son malheur, une invitation de la reine Christine de Suède. Il mourut à Stockholm le 11 février 1650, dans sa cinquante troisième année, victime du terrible hiver suédois.

Jean BERNHARDT

Descartes et l'argumentation philosophique. Sous la dir. de Frédéric Cossuta. Paris, Presses universitaires de France, 1996. $15 \times 21,7,245$ p. (L'Interrogation philosophique).

Les auteurs de cet ouvrage tendent à une étude de l'argumentation philosophique devant aboutir à une théorisation de celle-ci. Descartes leur fournit l'occasion de mettre à l'épreuve certains concepts, certains outils ou certaines hypothèses qu'ils ont élaborés. Frédéric Cossuta affirme ainsi en introduction qu'il ne s'agit pas de présenter un autre Descartes mais plutôt une autre façon de lire Descartes, en tenant compte du renouvellement récent des disciplines interrogeant langage et discours. Répondant à une question critique, il dégage les conditions de possibilité d'une 
théorie de l'argumentation philosophique, qui ne doit pas se limiter à l'horizon de la démonstration philosophique où la rationalité serait par excellence à l'œuvre, mais bien plutôt s'ouvrir à ce qui la constitue également comme discours, à savoir les aspects rhétoriques, stylistiques, etc., aspects trop longtemps oubliés. Ainsi démontrer peut parfois requérir une démarche de persuasion; à cet égard, le choix de Descartes paraîtra d'autant plus intéressant qu'il semble introduire une rupture avec les procédés rhétoriques qu'utilisaient les scolastiques. Plutôt que d'opposer, à l'instar de toute une tradition, démonstration et argumentation, il s'agira de voir comment elles concourent et permettent l'élaboration d'un discours philosophique. F. Cossuta propose ainsi une histoire des tentatives d'analyse de l'argumentation et montre les limites de celles-ci en ce qui concerne une théorie de l'argumentation philosophique, tout en reconnaissant certains héritages. Par là, il affirme que Descartes a choisi « une utilisation non rhétoricienne de la rhétorique » - là aussi résiderait son originalité - et que tous les phénomènes argumentatifs qu'il utilise doivent être pensés corrélativement au contenu doctrinal qu'il déploie.

Alban Bouvier, en se situant dans le prolongement d'une sociologie de la connaissance, essaie de comprendre comment Descartes, combattant le scepticisme, a produit «malgré lui » quelque chose que l'on peut qualifier d'éthos sceptique. Il reprend, comme critère de cohérence textuelle, deux règles qui vont ensuite guider son propos - la règle de progression et la règle de redondance - qu'il confronte au texte des Méditations: ainsi le processus de découverte liée à la voie analytique témoignerait de l'usage de la règle de progression. Qu'en est-il donc de la question du scepticisme? Après une étude très attentive du texte et de ses variations, il conclut provisoirement que les interprétations sceptiques ont quelque raison d'être d'un point de vue argumentatif puisque Descartes n'indique jamais réellement l'existence d'une progression (et ce par des marqueurs textuels) qui autoriserait les reformulations qu'il fait subir au concept de scepticisme. Peut-on dès lors conclure à une incohérence dans le texte? C'est le concept de polyphonie qui permet d'échapper à la menace sceptique dans la mesure où l'on peut supposer que les Méditations dressent une «microsociologie de la communauté philosophique ». L'analyse de l'argumentation témoigne donc ici de sa fécondité. En étudiant tous les ressorts de la discursivité cartésienne, A. Bouvier montre que les «mésinterprétations " sont contenues comme possibilité dans le texte cartésien, puisque Descartes a transgressé ou masqué certaines normes de communication nécessaires, normes liées aux règles déjà citées.

Dominique Maingueneau, dans un horizon d'analyse du discours, veut montrer qu'une œuvre philosophique ne se réduit pas à ses contenus doctrinaux mais qu'elle construit un « univers de sens à travers son énonciation même ». Reprenant le concept aristotélicien d'éthos, il veut montrer que tout discours est associé à une vocalité, à un ton, donc à un corps qui joue le rôle de garant. Ainsi l'éthos est partie prenante du dispositif énonciatif et n'est pas séparable de la scénographie du discours, c'est-à-dire d'une scène de parole que le discours institue. Il s'agit donc de faire de «l'événement énonciatif le foyer autour duquel s'organise le discours philosophique ». Analysant le Discours de la méthode, D. Maingueneau affirme qu'il s'agit d'un texte « second » car ce n'est pas le lieu où se constitue le noyau doctrinal du cartésianisme, mais fondateur parce qu'il est un monument stylistique et que, de ce fait, il fut à l'origine d'un développement historique du cartésianisme. Ainsi, 
l'usage des métaphores du cheminement témoigne de la réversibilité entre ce qui est dit et la manière de le dire. On pourrait parler d'interaction. Il en est de même de l'usage du français qui participe du contenu qu'il véhicule, celui de l'éclairement, celui des "lumières » pourrait-on dire; tous ces éléments permettant ce que D. Maingueneau qualifie d'institution de discours à travers une scène de parole. Grâce au concept d'éthos, on peut penser l'identité d'une philosophie qui demeure en droit transmissible à travers des énoncés généraux, mais qui reste particulière et irréductible, sans doute, dans sa manière de les dire.

F. Cossuta, toujours dans le cadre d'une analyse du discours, veut étudier les opérations formant la scène philosophique qui structurent l'énonciation de la discursivité philosophique. Il s'agit d'analyser les procédés de mise en discours qui relient l'œuvre à son extériorité et à son intériorité. La légitimation porte ainsi sur la relation de l'acte énonciatif et des énoncés à la situation d'énonciation; l'argumentation sur la relation entre énonciateur et coénonciateurs à travers un philosophème; la validation concerne la structuration interne de la doctrine. Il utilise également les termes d'institution philosophique (" manière dont le discours tend à s'instituer dans le champ social ») et d'instauration philosophique (« mouvement par lequel une philosophie construit un corps doctrinal autonome »). F. Cossuta propose dans ce cadre un parcours, très rigoureux et riche, retraçant la constitution de la doctrine cartésienne : Descartes cherche d'abord à obtenir la légitimation de son propos sans faire œuvre, en utilisant des stratégies de diffusion doctrinale biaisées, tel le larvatus prodeo, on s'intéressera à cet égard à la Correspondance; avec le Discours de la méthode, la volonté de convaincre se fait jour à travers le récit de vie, exemplaire de la méthode, dès lors il s'agit d'argumentation; les Méditations métaphysiques explorent, quant à elles, de nouvelles modalités d'adhésion — donc de validation -, l'ordre des raisons et le mode d'exposition; mais elles appellent des réécritures qu'il faudrait analyser : Réponses aux Objections et Principia. Ainsi Descartes semble investir différents genres, en faire des modes d'expression adéquats de sa doctrine, ou inadéquats, comme l'indiquent les tentatives avortées d'exposition du cartésianisme, parce qu'il n'a pas réussi à conduire simultanément instauration et institution. Il s'agit ici d'un article programmatique dont on attend la suite, surtout pour ce qui concerne les Principia.

Christophe Giolito propose d'analyser, du point de vue de l'histoire de la philosophie, le texte des Passions de l'âme et sa lecture leibnizienne dans le De affectibus dont il traduit quelques articles en appendice. Il s'agit de comparer des situations d'énonciation différentes «pour mettre en valeur le rôle différentiel des opérateurs d'argumentation ». Après avoir décrit les situations d'énonciation respectives des auteurs, qui sont autant de conditions de possibilité des textes, il propose une comparaison minutieuse pour aboutir à la conclusion suivante; si le texte de Leibniz ne comporte que peu d'indices textuels, cela renvoie à sa théorie génétique de la définition et donc l'argumentation se voit régie par le contenu doctrinal, tout autant qu'elle le régit; l'objet n'est pas extérieur chez Leibniz à la conceptualité qui le produit, à l'écriture qui le porte et l'argumentation devient dès lors un défi à soimême. Le Traité des passions, en revanche, témoigne de la volonté de concilier les différentes dimensions de l'élaboration théorique, où l'argumentation permet de différencier plutôt que de réunir sous une même définition. 
Enfin, Jean-François Bordron, dans l'horizon d'une sémiotique, se propose d'étudier les contraintes génériques et l'argumentation à l'occasion de la question de la triplicité des preuves de l'existence de Dieu dans les Méditations. Selon lui, la triplicité des preuves serait à lire dans la nécessaire triplicité d'une structure d'appréhension (qu'il analyse en termes kantiens : synthèses d'appréhension, de reproduction et de recognition, rapportant celles-ci à l'infini) et non dans les différents ordres qui autorisent les démonstrations. Les trois preuves partant d'un même constat (j’ai en moi l'idée d'un être souverainement parfait) veulent montrer que ce fait est tel, que l'idée de Dieu n'est pas matériellement fausse. Les preuves seraient alors une saisie du contenu de l'idée, du même contenu dont elles proposeraient une schématisation.

Ainsi si certains des auteurs de ce volume revendiquent pour l'argumentation philosophique un statut autonome (Cossuta et Maingueneau), les autres préferrent lui reconnaître le statut d'objet pour des disciplines déjà constituées. Il nous semble, en effet, qu'il y a un intérêt à analyser toutes les procédures par lesquelles un discours philosophique se dit, et cela au risque de l'incohérence - en ce sens cet ouvrage nous semble important; cependant, ne doit-on pas mesurer la finesse de Descartes qui rejetait la rhétorique en tant que discipline et non en tant que pratique?

Elsa Rimboux

Vincent Jullien, Descartes, la Géométrie de 1637. Paris, Presses universitaires de France, 1996. 11,5 × 17,5, 128 p., bibliogr. (Philosophies, 76).

Il faut bien reconnaître que la Géométrie est un texte peu lu et peu connu, relativement à l'ensemble du corpus cartésien. C'est pourtant un essai central à la fois dans le système de Descartes et dans l'histoire des mathématiques. Il faut donc faciliter l'accès de ce traité difficile.

L'ouvrage de Vincent Jullien se présente comme un précieux guide de lecture. Partant du principe qu'en « abordant la lecture de l'Essai de 1637, on doit prendre conscience du regard que l'on y porte » (p. 54), l'auteur s'attache à fournir à son lecteur, d'une part un réseau dense d'informations sur la place de Descartes dans l'histoire des mathématiques et sur la place de la Géométrie dans l'Euvre, d'autre part une étude suivie des trois livres du traité de 1637, enfin et surtout un axe de lecture fondé sur une discussion des positions actuelles quant à ce texte. Dans le plan de l'ouvrage, c'est la décision de l'axe de lecture qui constitue l'articulation entre l'élaboration de l'appareil critique et l'étude détaillée de l'ceuvre. On aura compris que le choix interprétatif n'est pas arbitraire mais qu'il se fonde sur un examen de trois horizons de lecture : celui fourni par l'histoire des sciences, celui fourni par le corpus cartésien, et celui fourni par l'état actuel des recherches sur la Géométrie.

Considérant que l'Essai de 1637 traite de deux grandes catégories d'objets, à savoir les équations et les courbes, le principal problème d'interprétation, que met bien en évidence l'auteur, est le suivant : " [...] de ces deux grandes catégories, quelle est celle qui organise, qui tient le premier rôle dans la Géométrie? Les 
courbes construites (ou constructibles) ou les expressions algébrique? » (p. 57). C'est-à-dire que si une lecture évaluatrice du point de vue de l'histoire des sciences, quant aux mérites, aux apports, aux erreurs et aux manques de la Géométrie est nécessaire, elle est néanmoins insuffisante, car «elle peut se passer de l'examen et de l'élucidation de l'organisation globale de l'Essai — du "fil de Thésée" - qui demeurera cachée » (p. 55). Il importe donc avant tout de prendre position en faveur d'une lecture " algébriste » ou d'une lecture " constructiviste ". Les deux positions s'avèrent insuffisantes. La première est, par exemple, celle de l'historien des sciences Enrico Giusti. La seconde est, par exemple, celle défendue par Henk Bos.

Voici l'énoncé de la décision interprétative de l'auteur qu'il va falloir ensuite détailler et étayer en examinant la première partie de l'ouvrage : « Doit-on renoncer à l'exploration puisque ni le versant "primat du géométriquement constructible", ni son opposé "primat de l'expression algébrique" ne nous conviennent? Peut-être pas; peut-être suffit-il de suivre Descartes dans une de ses thèses les plus fortes de l'Essai, et l'une des moins bien établies pourtant. Une courbe est constructible par instrument légitime lorsqu'elle admet une équation algébrique» (p. 64, c'est nous qui soulignons).

L'auteur ne se livre pas à la construction mathématique d'une preuve de " complétude ». La lecture se veut surtout proche de Descartes, et, non seulement, dans l'œuvre de ce dernier, une telle preuve, et même l'idée d'une telle preuve est absente (et pour cause!), mais aussi, dans l'esprit de ce dernier, "cette absence est — en principe - sans importance, ou sans dommage : la certitude des raisons générales méthodiques et philosophiques confortée par la maîtrise d'une série d'arguments-exemples, où la vérité se donne à voir géométriquement puis algébriquement vêtue, suffit » (p. 51). La cohérence du texte va être mise en évidence au fil d'une argumentation serrée qui a pour fin de replacer l'Essai en son contexte. Il s'agit principalement de mettre en évidence ce qui fait l'originalité de la géométrie de Descartes.

La cohérence de l'Essai ne peut être comprise adéquatement que si l'on prend la mesure de cette originalité. Il faut établir les points suivants, ce que l'auteur s'emploie à faire dans la première partie; 1) le projet cartésien est contenu dans les Regulae et dans le Discours de la méthode et est fondé sur une critique du paysage mathématique qui est familier de Descartes : d'une part, la géométrie des Anciens, d'autre part, l'algèbre des Modernes; 2) la Géométrie, contrairement à ce qu'affirment certains commentateurs, comme Ferdinand Alquié, n'accuse aucun recul par rapport à l'enjeu programmatique des Regulae. Croire qu'il s'agit d'un recul, c'est tout simplement faire un contresens sur la mathesis universalis, qui ne s'identifie aucunement, si l'on lit bien Descartes, avec la géométrie ou l'algèbre. Le programme des Regulae est celui de la mathesis universalis. Mais ce dernier ne se résume pas à la partie de ce traité qui correspond aux «questions parfaitement posées » de la géométrie : «[...] la Géométrie n'est évidemment pas un traité de mathesis universalis. Pourtant, si l'on se réfère à la partie rédigée des Regulae, à l'examen des questions parfaitement posées, il faudra être beaucoup plus positif. La Géométrie de 1637 réalise effectivement [...] cette partie-là du programme général » (p. 47); 3) la correspondance entre lignes et symboles ne se comprend qu'à partir des deux premiers points : «La Géométrie de Descartes est bel et bien bâtie sur la 
grande doctrine eudoxo-euclidienne des grandeurs continues, mais elle parvient à y greffer un élément neutre et une multiplication » (p. 74). En effet, d'une part, la géométrie des Anciens est vraie, d'autre part, l'algèbre des Modernes constitue, une fois la notation allégée, un puissant auxiliaire pour l'imagination, afin de parcourir en un seul regard, les « longues chaînes de raisons » des Regulae. La théorie des proportions est vraie en ce qu'elle assigne à la géométrie un attribut incontestable de l'étendue, à savoir la grandeur continue. Mais les mathématiques grecques se présentent comme morcelées, c'est le projet de Descartes pour la science qui doit s'appliquer en faisant des mathématiques un paysage unifié. Pour cela, l'algèbre est une auxiliaire précieuse. V. Jullien cite, par ailleurs, un autre des Essais de $1637:$ la Dioptrique (voir Euvres de Descartes, éd. Charles Adam et Paul Tannery, nouv. présentation par Bernard Rochot et Pierre Costabel, 11 tomes, Paris, Vrin, CNRS, 1964-1974, t. VI, p. 112-113) pour argumenter son interprétation de l'imagination cartésienne comme «[...] expression, mise en rapport réglée entre l'objet et l'esprit » (p. 17).

De tout cela, il résulte que la géométrie cartésienne n'a pour objet, au sens fort du terme, ni les équations, ni les courbes, que celles-ci ne sont qu'un « revêtement », une «parure », pour la « vérité », l'unité, c'est-à-dire l'ordre et la mesure. La cohérence de la Géométrie est donc celle de la mathesis universalis, non pas en ce que la géométrie cartésienne traite de l'ordre et de la mesure, mais en ce que, appliquée à un secteur, l'étendue, elle en déploie tout ce que l'on peut en dire légitimement, c'est-à-dire selon les préceptes de la méthode. Ainsi, l'examen du problème de Pappus est-il bien mis en évidence par l'auteur, dans la seconde partie de son ouvrage, comme une occasion pour Descartes d'ordonner le domaine des courbes par rapport à un problème de lieux : il y a des problèmes linéaires, plans, solides, sursolides, et la résolution se fait par un parcours des degrés de difficulté. L'auteur note, par ailleurs, une erreur de Descartes dans l'affirmation un peu précipitée de l'exhaustivité de l'examen du problème de Pappus par rapport à l'ensemble des lignes courbes.

D'emblée, le cadre dessiné pour les mathématiques par Descartes est limitatif. D'une part, il s'interdit de réévaluer la validité générale des Éléments d'Euclide : « La position cartésienne consistant à fonder la géométrie sur des énoncés intuitivement acquis s'oppose aux efforts à peu près contemporains de Roberval et de Pascal visant à la fonder selon une méthode quasi axiomatique » (p. 11). D'autre part, il limite l'algèbre en barrant l'accès à une théorie des nombres, par l'interprétation qu'il fait de cette nouvelle science : « [...] les mathématiques de Descartes [...] évitent la considération des nombres qui sont en quelque sorte les grands absents de cette science » (p. 72).

Descartes est conscient d'introduire des bornes au connaissable géométriquement, en en rejetant, par exemple, les courbes « transcendantes », c'est-à-dire celles qui ne sont pas « constructibles par instruments légitimes » (les compas cartésiens, l'auteur renvoie à ce sujet aux travaux de Michel Serfati). Mais, en citant la lettre à Debeaune du 20 février 1639 (p. 126), Vincent Jullien montre bien qu'il ne s'agit pas chez Descartes d'une incapacité technique à manier les méthodes d'indivisibles, mais bien d'un refus essentiel de méthodes qui utilisent une notion inconnaissable comme l'infini, ainsi pour la quadrature de la cycloïde, ainsi pour le problème de Debeaune, ainsi pour les imaginaires. La limitation ne doit donc pas être prise 
comme un manque, mais, ainsi conclut l'auteur, "voici comment sont gardées les frontières d'un pays qu'il [Descartes] entend avoir exploré en entier» (p. 126).

Christophe Alsaleh

Frédéric de Buzon et Vincent Carraud, Descartes et les « Principia » II. Corps et mouvement. Paris, Presses universitaires de France, 1994. 11,5 × 17,5, 128 p., bibliogr. (Philosophie, 52).

La collection «Philosophies » des Presses universitaires de France, dont la Revue de synthèse a eu l'occasion d'évaluer l'excellence, fait paraître un volume consacré à la physique de Descartes. Le texte de la deuxième Partie des Principia, donné récemment à l'agrégation de philosophie, est en effet un texte relativement méconnu, à propos duquel on se contente, en général, de quelques banalités que l'on estime être le dernier mot au sujet de la physique cartésienne. Or, de « physique cartésienne », il n'est pas question ici; le propos, de façon très heureuse et salutaire, analyse de façon suivie, linéaire, le texte même de Descartes. Frédéric de Buzon et Vincent Carraud entendent moins donner une voie d'entrée à la lecture de Descartes que «préparer le terrain pour de nouveaux ouvrages », constatant que les Principia n'ont que très peu bénéficié du « renouveau des études cartésiennes ». En proposant de lire un texte peu maîtrisé, tant par la culture générale des philosophes que par la recherche elle-même, les auteurs répondent parfaitement à l'orientation première de la collection qui est d' "'élargir le domaine des questions et des textes habituellement considérés comme philosophiques et d'en ouvrir l'accès à un public qui en a été tenu écarté jusqu'ici ». Mais en réservant (voir l'Avant-propos) la lecture de leur ouvrage à ceux qui " ont déjà quelque connaissance de la pensée de Descartes ", ils infléchissent sensiblement une autre finalité de cette précieuse collection, celle qui la destine à la diffusion, chez les non-spécialistes, d'un savoir qui leur était interdit. Or il apparait clairement, lorsque l'on en achève la lecture, que chacun peut aisément tirer profit d'un tel travail. S'il faut effectivement avoir le texte de Descartes à portée de main, ce ne sont pas seulement les spécialistes qui doivent se réjouir de l'existence d'un tel outil de travail.

F. de Buzon et V. Carraud évoquent tout d'abord la naissance des Principia en insistant sur leur statut de Summa philosophice pensée sur le modèle des manuels anciens (par ex., celui d'Eustache de Saint Paul). Les Principia forment la seconde partie d'une architecture d'ensemble de la philosophie, comprenant en outre les Specimina philosophiae (Discours de la méthode et Essais). Dans une présentation rapide, les auteurs font le point sur le style propre des Principia, récusant l'approche conventionnelle qui oppose les analytiques Meditationes et les synthétiques Principia.

La difficulté que soulève la traduction française est très classiquement repérée dans le fait que si une traduction, même approuvée par l'auteur, reste seconde et n'interdit aucun travail ultérieur, le texte de 1647 possède par ailleurs (voir les articles 46 à 52 et les modifications notables des règles du choc) la marque d'un infléchissement et peut aussi prétendre à la valeur d'un original. Abordant la ques- 
tion des rapports de la physique (une analyse plus longue est rejetée, par les auteurs, à la fin de l'ouvrage, lorsqu'il s'agit de commenter l'article 64 des Principia $I$ ) et de la métaphysique, de Buzon et Carraud mettent en avant l'impossibilité de penser le passage de cette dernière à la première sous le régime de la «déduction ». Cette indication est par ailleurs reprise in fine (p. 124) par la mention d'une nécessaire "brève histoire des phénomènes ", c'est-à-dire la prise en compte par Descartes de ce que les auteurs appellent la grande «productivité des lois» ou encore un « moment empirique essentiel ». Le problème de la déduction est central et on peut regretter qu'il ne soit ici que l'objet d'une petite mise en garde (et, à l'occasion, d'une critique de l'ouvrage de Michio Kobayashi, La Philosophie naturelle de Descartes, Paris, Vrin, 1993). L'élucidation exacte du mode de production des principes de la physique (les auteurs affirmant que la métaphysique ne fait que "garantir » ce que la physique considère déjà comme un principe, p. 32) ainsi que la délimitation lexicale des propositions visant ce que Dieu « fait ou maintient » (principes, fondements, p. 30) et ce à quoi la nature se soumet (lois et règles) en vertu de l'action même de Dieu, permettraient de revenir sur certains usages répandus en histoire et philosophie des sciences. Prenons pour exemple cette séparation omniprésente entre, d'une part, une « méthodologie » cartésienne qui « déduirait » a priori les lois de la physique en fonction des principes de conservation par lesquels on décrit l'action de Dieu et, d'autre part, la science active de Descartes (ou, à défaut, de ses successeurs en rationalisme). F. de Buzon et V. Carraud, en condamnant l'idée qu'il y ait une déduction métaphysique des principes, veulent sans doute, à juste titre, remettre en cause une conception trop hégélienne et a priori du déduire dans la compréhension de ce qui se joue chez Descartes. Cette mise au point, qui n'est pas totalement effectuée ici (les limites de la collection prévenaient toute possibilité de trancher) permettrait de combattre un certain discours, très irritant et trop lointain, sur Descartes. Il n'en est pas moins vrai que, même si l'on accorde sans discussion à Jean-Luc Marion la thèse d'un redoublement métaphysique (Sur le prisme métaphysique de Descartes, Paris, Presses universitaires de France, 1986; cité p. 32) des principes physiques ainsi que l'idée de « principes de principes », les «principes » sont avant tout pertinents dans le champ de la métaphysique (la physique s'accommodant au mieux des lois premières ou secondes) et l'on voit aisément qu'ils fonctionnent selon une règle d'exclusion : ce qui n'est pas fondé en raison (par la métaphysique) n'est pas principe de physique, simple contraposée de cette proposition selon laquelle la métaphysique «assure ce que la physique considère déjà comme un principe » (p. 32). Ce que la physique « considère », tel est le cercle vicieux, comment pourrait-elle le tenir d'elle-même? Cette vie autonome de la physique et le libre décret par lequel elle pense au préalable (via la mesure et l'« histoire des phénomènes ») ses principes est vérifiée chez un Huygens : l'est-elle chez Descartes?

Une autre indication de cet ouvrage tient en l'affirmation, rapide mais ferme, que c'est vers Leibniz qu'il faut se tourner pour comprendre la nature exacte de la « liaison cartésienne constitutive de la physique à la métaphysique ». C'est que Leibniz n'entend pas critiquer la liaison elle-même, mais ses termes. Les noms de " Huygens, Spinoza, Malebranche, Fontenelle ou Newton » sont cités comme des sources secondaires de cette réception/évaluation. Demeurant d'accord avec le propos général, signalons toutefois que le projet qui consiste à éclairer les Principia ne peut 
envisager la lecture de ces auteurs comme simple consultation opportune (p. 33). Dans le cas de Huygens, on dispose d'un observateur particulièrement attentif aux fausses critiques. Leibniz, procédant par mauvaises identifications, n'a pas été à même de comprendre la pertinence du concept cartésien de conservation de la quantité de mouvement dont il croit donner le sens à partir de sa propre compréhension de la conservation globale de la force. C'est la connaissance d'un point de vue comme celui de Huygens - voyez sa critique de la Brevis demonstratio - qui permet de comprendre adéquatement une grande partie de l'édifice conceptuel des Principia II (voir Jean-Pierre Séris, Machine et communication, Paris, Vrin, 1987, p. 239 sq., ainsi que Michel Fichant, La Réforme de la dynamique, Paris, Vrin, 1994, «Introduction», passim. Nous nous appuyons aussi sur un cours de M. Fichant prononcé à Paris-I, hiver 1992).

L'analyse linéaire est divisée en trois moments. Le chapitre "Les corps » commente les articles 1 à 23 . Le chapitre «Le mouvement » élucide les articles suivants, jusqu'à l'article 63. L'ultime chapitre «Physique et mathesis » traite du seul article 64 et joue le rôle d'une conclusion générale articulée à la question de la nature de la "physique ", entre pureté ou abstraction de la mathesis et concrétude que le mot même de "physique " laisse supposer. Le découpage en mouvements successifs est toujours en quelque façon une thèse. On peut être surpris de cette architecture qui sépare les articles 1 à 23 (analyse des conséquences de l'équivalence de la chose corporelle à la chose étendue) des articles 24 à 35 (considération de la division infinie de la matière introduite par le mouvement, article 24 , qui est seulement cause de la diversité). Cette seconde série peut en effet être considérée comme étant du même ordre que la première, dont elle ne fait que déduire les conséquences. De ce point de vue, l'analyse du mouvement ne commence authentiquement qu'avec l'enchaînement déductif qui va de Dieu comme cause première (article 36) vers les règles particulières du choc (articles 45 à 52) en passant par l'énoncé des trois causes secondes (ou lois de la nature, 37 à 44). C'est l'article 36 qui permet de rendre compte d'un changement de ton et de point de vue dans l'économie des Principia II. Le mouvement est certes abordé avant cet article 36, mais c'est seulement d'un point de vue modal. Sans doute la présentation adoptée par F. de Buzon et V. Carraud trouve-t-elle sa justification dans la volonté de ne pas accorder une importance trop grande à la conversion du regard de Descartes, passant de la considération du mode et de la substance vers celle de l'action divine en tant que cause. Il est vrai que l'on met alors en avant un noyau de propositions a priori accréditant en apparence l'idée d'une « déduction » elle-même $a$ priori de la conservation de mouvement à partir de l'immutabilité de Dieu comme cause première (ce qui n'est pas le cas).

Pour étrange que ce découpage puisse paraître, il n'invalide en rien la pertinence de l'interprétation précise, travail remarquable et clair où la compréhension des concepts de Descartes (re-situés le plus souvent dans leur généalogie) se trouve facilitée, même si elle ne devient pas pour autant aisée. Voilà un ouvrage essentiel, propédeutique autant que noyau autonome de sens, qui manquait au paysage universitaire français. 
Édouard Morot-Sir, La Raison et la grâce selon Pascal. Préf. de Jean Mesnard. Paris, Presses universitaires de France, 1996. 13,7 × 21,5, xıx-273 p. (Écrivains).

Les enquêtes philosophiques exposées dans cet ouvrage peuvent, à juste titre, être considérées comme des «méditations pascaliennes ", en acceptant que ce soit le sujet de la méditation et non le mode de réflexion utilisé qui puisse être rapporté à Pascal. En effet, l'ensemble des sept textes détonne dans le paysage de la recherche contemporaine. L'auteur préfere nettement écrire « avec » Pascal qu' " au sujet de » ou «dans » Pascal, ce qui serait tout à fait recevable si ce projet était pleinement assumé. Au contraire, le livre d'Édouard Morot-Sir oscille entre la réflexion intimiste et personnelle et la thèse universitaire, sans que l'un et l'autre de ces genres ne tirent profit de leur cohabitation au sein d'une même étude. L'érudition déployée est très souvent ancienne, les thèses avancées assez peu originales. L'utilisation faite des textes de Pascal est loin d'être irréprochable, comme c'est le cas notamment au début du chapitre iv, au moment de l'exploration des " champs sémantiques " des Pensées, où l'on peut lire un lexique des termes les plus employés par Pascal, donné sans ordre ni méthode. Un tableau des occurrences placé en annexe aurait été plus simple et plus efficace.

Le titre de l'ouvrage ne donne qu'une idée trop vague de son contenu et reflète assez mal le projet d'ensemble. De tous les essais qui composent l'ouvrage, les plus importants sont ceux consacrés à l'analyse du langage chez Pascal, que ce soit au niveau de la propre réflexion de Pascal sur le sujet ou encore sur les enjeux théoriques de la rhétorique pascalienne. Les deux textes proposant une lecture « pascalienne " d'auteurs contemporains importants - Wittgenstein, Beckett, Bataille, Sartre et Camus - s'écartent du ton, voire même du propos, de l'ouvrage. Il n'était peut-être pas vraiment nécessaire ni pertinent de les publier dans ce volume. Endehors de leur facture pour le moins étrange, l'ensemble des articles offre une bonne synthèse sur ce problème classique mais incontournable du langage et de la rhétorique de Pascal, qualifiée par Morot-Sir d' « épistémologie de la foi », problème qui, il est vrai, est trop souvent abordé de biais par les commentateurs, que ce soit par celui de la philosophie, du style littéraire ou encore par l'étude du thème de la " figure " chez Pascal. Si le problème du langage est bien le cour de l'ouvrage, on aurait tort cependant de penser que la rhétorique pascalienne est étudiée dans ses rapports avec les finalités de la tradition thétorique classique : convaincre, plaire et émouvoir. Pressé d'en venir aux questions théologiques, Morot-Sir délaisse les questions du style pour en arriver plus vite au thème de la grâce.

En guise d'introduction à ces études, Morot-Sir commente le «conflit métaphysique et religieux » qui oppose jésuites et jansénistes dans la France classique. Toute la question de la dualité du corps et de l'esprit est pensée dans les termes imposés par l'anthropologie christocentrique de l'Áge baroque et classique. Mais l'opposition construite par Morot-Sir demeure inexploitée. Tout se passe comme si l'auteur préférait opposer deux visions du monde - jésuite et janséniste - en juxtaposant leur doctrine respective, et sans chercher à repérer ni commenter les enjeux de ces oppositions. Il en va de même pour les parallèles, repris de Maurice Blondel, rapidement expédiés entre les techniques ignatiennes des Exercices spirituels et le 
discours apologétique de Pascal au sujet de la méthode employée pour faire « ployer les esprits ". Bien sûr, les recherches sur ces sujets ne manquent pas, mais alors pourquoi ne pas passer rapidement de la synthèse globale du conflit à un exposé détaillé des divergences entre jésuites et jansénistes sur la question du langage. Le chapitre VI, sur la " pédagogie janséniste de la parole ", ne nous renseigne pas plus sur la pédagogie supposée adverse, celle pratiquée dans les écoles jésuites. MorotSir souligne les changements majeurs effectués quant aux choix des moyens employés et des buts visés par la rhétorique janséniste, celle de Nicole et celle de Pascal, où l'art du discours prépare le sujet à la conversion. Le langage humain est et demeure mystérieux dans l'attente de la foi. Il aurait été intéressant de comprendre plus précisément la rupture de ton entre l'éloquence, discours de l'honnêteté, et la pédagogie janséniste, dont l'honnêteté n'est qu'une étape. Même dans l'article central du livre, le chapitre iv intitulé « La raison selon Pascal. Géométrie du sens, calcul de la parole ", Morot-Sir préfêre un dialogue avec Descartes, en raison de son éducation chez les jésuites du collège de La Flèche, et Leibniz, ce qui lui permet de faire appel à toute la batterie des concepts clés pascaliens contre les systèmes de ces deux auteurs, mais ne précise ni n'éclaire davantage l'exploitation par Pascal des échanges entre les différents modes du discours.

Un thème important de l'ouvrage est celui de la démonstration des propriétés herméneutiques de l'analyse pascalienne du langage, et ce, dans les rapports entretenus entre l'ontologie cartésienne et l'apologétique. Le travail réflexif de l'égologie n'inscrit pas le sujet dans un rapport de réalité avec le monde, puisque ce rapport n'est pas d'abord celui entre l'homme et Dieu. La rationalité ne fait que confirmer la vérité divine: son rôle est toujours secondaire. La vérité divine seule importe et autorise l'affirmation de l'existence. Le rapport au moi, si dépouillé soit-il, ne vaut pas plus que la description des «qualités » de l'homme. Mais la complexité de l'égologie cartésienne est tellement diminuée par Morot-Sir que Descartes n'est plus qu'un homme de paille offert en pâture aux critiques de Pascal. Du même coup, l'intérêt philosophique de l'opposition construite entre Descartes et Pascal est atténuée.

L'analyse de Morot-Sir a le mérite de replacer la question du langage au centre de l'anthropologie théologique de Pascal, en interrogeant, par exemple, la simultanéité sémantique des termes du langage ordinaire, signes à la fois du monde temporel et de l'intemporel, à partir du dualisme de l'âme et du corps. C'est par le langage seul que l'homme échappe à la finitude et parvient à transformer une part de son être en langage divin : la prière. La question de la présence nécessaire, dans le langage de l'homme, de la vérité divine comme langage absolu, est assez finement commentée par l'auteur. L'homme étant un être fini dans le désir de l'infini, l'ouverture qui fait du langage humain bien plus qu'un simple mode clos de relation est celle des figures du désir d'infini des hommes. C'est dans cette brèche que se transforme le désir d'infini en nécessité, si la grâce opère et accomplit la métamorphose du langage en prière. Mais la dualité propre au langage, porteur à la fois de la contingence et de l'universalité divine, ne peut être résolue au moyen de l'union parfaite des contraires, comme celle rêvée par Leibniz. Chez Pascal, l'art de persuader ne délivre pas l'homme de son ignorance de la vérité, mais le prépare à se délivrer des erreurs commises par la machine corporelle, et ce, parfois au moyen même de ces erreurs, notamment par le truchement de l'imagination. La pédagogie de la parole est alors 
un exercice protreptique dont l'objectif est l'« uniformité d'esprit entre le moteur qui inspire nos passions et celui qui permet les résistances à nos passions » (Blaise Pascal, Euvres complètes, éd. Louis Lafuma, Paris, Seuil, 1963, p. 284). Ce moteur permettant les passions n'est nul autre que Dieu, qui transforme le désir de grandeur en recherche de la vérité.

Pour conclure, s'il faut insister sur la valeur et la richesse de nombreuses thèses de l'ouvrage, on regrettera surtout les choix méthodologiques de l'auteur et surtout la valeur inégale des textes réunis ici. On ne peut reprocher à l'auteur une connaissance insuffisante du corpus pascalien ni de n'avoir pas su exploiter toute la richesse des textes moins fréquentés comme la correspondance ou les traités scientifiques. En fait, c'est l'efficacité du mode rhétorique choisi par l'auteur qu'il est possible d'interroger. Ce problème est particulièrement visible au moment où l'auteur tente de produire une synthèse en conclusion d'une réflexion. C'est le cas pour la thèse centrale du livre, où Morot-Sir néglige de pousser plus avant sa compréhension du passage du langage ordinaire à la prière dans le texte même de Pascal. Il y aurait pourtant beaucoup à dire sur les variations de style à l'œuvre chez Pascal, même si le discours brisé des Pensées empêche de concevoir une volonté derrière le rythme propre à l'enchaînement des fragments. Il faut espérer une étude plus concluante sur ces thèmes.

\section{Christian NADEAU}

Catherine Chevalley, Pascal. Contingence et probabilités. Paris, Presses universitaires de France, 1995. 11,5 × 17,5, 128 p., bibliogr. (Philosophies, 57).

Contrairement à ce que le titre de l'ouvrage pourrait laisser penser, Pascal. Contingence et probabilités ne propose pas une nouvelle étude de la mathématisation du hasard qui, inscrivant cette découverte parmi les innovations scientifiques de Pascal, laisserait de côté toute considération philosophique sur la contingence. Le but de l'auteur étant d'établir un lien entre philosophie et science, son ouvrage permet au contraire, en traitant de façon générale la question de la contingence, de faire une synthèse de la pensée de Pascal à partir de textes en apparence aussi différents que les Pensées, le Traité des liqueurs, les Discours sur la condition des Grands, la Préface au Traité du vide, les Lettres à Le Pailleur et à Fermat, en évitant remarquablement un écueil qui se présente à toute approche globale des textes de Pascal : la reconstitution architectonique de la pensée d'un homme dont l'écriture et la conception de la connaissance furent pourtant à l'opposé de la construction systématique.

Pour Catherine Chevalley, Pascal fut non à la fois mais tour à tour et selon des modalités différentes géomètre, physicien, philosophe et chrétien. Aussi, pour comprendre l'unité de sa pensée sans le transformer en penseur dogmatique, est-il nécessaire d'appliquer à son étude le concept central de sa propre pensée, la relation, en confrontant les différents domaines de sa pensée (mise en relation) et en 
identifiant le passage de l'un à l'autre à un changement de perspective (relativisation).

Le point de vue choisi est celui de la genèse d'une pensée qui se constitue progressivement sous l'impulsion de la critique de la philosophie cartésienne. Elle a lieu d'une part sur le front des mathématiques, où Pascal introduit un infini dissocié du sentiment naturel, concevable sous la forme d'un calcul opératoire, et d'autre part sur le front de la philosophie, où il définit la connaissance humaine comme naturellement incomplète, contingente, et relative à notre mode de perception.

La reconnaissance de la contingence et de la relativité du savoir autorise des innovations méthodologiques qui permettront le calcul des chances : passage de l'étude des essences à l'étude des relations, calcul par récurrence, application aux mathématiques du changement de point de vue, composition d'énoncés contraires. Ainsi naît une mathématique du hasard qui permet de lire l'argument du Pari, non comme un calcul mathématique de l'existence de Dieu, mais comme l'échec même de la lumière naturelle à en fournir la preuve.

La réunion du versant cartésien et épistémologique en une réflexion d'un seul tenant paraît parfois inaccomplie ${ }^{4}$, comme en témoignent les deux conclusions (correspondant aux deux parties de l'étude). Aussi est-il regrettable que l'importance du pyrrhonisme dans la conception pascalienne de la contingence ait été délibérément occultée ${ }^{5}$ : en lui accordant une place de choix, loin de contrevenir à la présentation non systématique de la pensée de Pascal, C. Chevalley aurait confirmé la pertinence de l'adoption de la relation comme concept central, et peut-être renforcé l'unité de son ouvrage.

Premièrement, on ne comprend pas bien comment l'auteur peut à la fois prétendre que Pascal se refuse de fonder métaphysiquement le savoir, et affirmer que le renoncement au projet philosophique cartésien n'est pas chez Pascal un renoncement sceptique, la seconde partie de l'ouvrage ayant pour objectif de nous en convaincre. Par rapport à une conception pascalienne de la science, Descartes n'est pas " tout sauf dogmatique » (p. 27). S'il est « inutile et incertain ${ }^{6}$ », c'est parce qu'il a échoué à surmonter l'épreuve du doute et que par conséquent la fermeté de ses principes est douteuse. Pascal doit au pyrrhonisme de considérer les principes (fondements) et les

4. L'extension à l'action humaine des travaux mathématiques sur les jeux de hasard demeure, par exemple, difficile à saisir, faute d'avoir été suffisamment développée dans ses implications philosophiques et morales.

5. Le scepticisme n'est pas vraiment ignoré (cf. p. 59 où est cité le fragment 170 dans l'édition des CEuvres complètes de Pascal par Louis Lafuma, Paris, Seuil, 1963). L'auteur concède (p. 9) que « les pyrrhoniens admettaient le hasard », et « qu'ils ne s'adonnaient pas aux calculs $»$. Est-ce une raison pour les juger indignes d'intérêt? Les sceptiques se refusent à donner à la fortune une expression rationnelle, mais, contrairement à ce qui est affirmé, ils la reconnaissent comme objet de penser. On peut en mesurer l'impact sur la conception pascalienne de la contingence.

6. Fragment 887 . 
fins comme des choses obscures ${ }^{7}$, d'estimer l'interprétation mathématique de l'expérience non révélatrice de l'essence de la chose ${ }^{8}$.

Deuxièmement, la référence à Montaigne dans l'élaboration d'une pensée de la contingence peut paraître aussi importante que la référence à Descartes. Ainsi, la description du milieu comme incertain et flottant (fragment 199), si elle peut évoquer Agrippa d'Aubigné ${ }^{9}$, constitue avant tout une compilation de plusieurs passages de l' «Apologie de Raymond Sebond » qui témoigne explicitement d'une conception sceptique de la réalité humaine où les deux critères potentiels de vérité, la raison ${ }^{10}$ et les sens, s'abusent mutuellement au sein du devenir. Le concept d' « interaction généralisée » introduit par C. Chevalley rassemble en fait les tropes d'Énésidème (sur la situation et les circonstances) subsumés par le sceptique Agrippa sous le mode plus général de la médiation, c'est-à-dire de la relation.

Troisièmement, les procédés qui permettent le calcul des chances sont également empruntés au pyrrhonisme : le changement de point de vue est un procédé sceptique de relativisation (qui permet de considérer les choses sous différents biais, en les mettant en situation ou perspective); le raisonnement par récurrence doit également beaucoup au scepticisme ${ }^{11}$ et au sceptique dont l'incertitude (forme subjective de la contingence où il est immergé), ne fait pas système, parce qu'elle s'applique à ellemême ${ }^{12}$.

Pour Pascal, nous ne connaissons en effet que ce à quoi nous avons rapport; mais c'est à condition d'en croire Pyrrhon et les pyrrhoniens. En somme, il n'aurait pas été inutile de rappeler que pour Pascal, si Descartes est dans l'erreur, c'est parce que "le pyrrhonisme est le vrai ${ }^{13}$ ".

Sylvia GrocanTI

7. Cf. fragment 199 : « La fin des choses et leurs principes sont pour l'homme invinciblement cachés dans un secret impénétrable. » Pascal, dans la tradition pyrrhonienne, distingue les phénomènes des adela. Il n'affirme jamais l'existence d'une nature du vide.

8. L'interprétation selon des conventions mathématiques est fiable, dans la mesure où elle supprime les représentations spontanées dont le contenu est toujours susceptible de varier, mais elle n'en est pas moins une construction de l'esprit.

9. Cf. p. 40.

10. La raison est tout particulièrement frappée d'impuissance. Pascal dresse un inventaire de ses handicaps largement inspiré de Montaigne.

11. La méthode par récurrence n'est autre que la reprise et l'extension au mathématique du passage à la limite tant usité par la Nouvelle Académie sur des questions de logique, et généralisé à toutes les questions de philosophie chez les pyrrhoniens.

12. Le sceptique n'est en effet pas certain que tout soit incertain (fragment 521), son doute s'incluant lui-même. Cf. l'Entretien avec monsieur de Sacy: «Il [Montaigne] met toutes choses dans un doute universel et si général, que ce doute s'emporte soi-même. »

13. Fragment 691. Cf. aussi fragment 131 : «On n'a qu'à voir leurs livres; et si l'on n'en est pas assez persuadé on le deviendra bien vite, et peut-être trop. » 
Johann Gottfried Herder, Dieu. Quelques entretiens. Trad. de l'allemand, prés. et notes par Myriam Bienenstock. Paris, Presses universitaires de France, 1996. $15 \times 21,5,194$ p., bibliogr., index (Théologiques).

Herder, dans son ouvrage, Dieu. Quelques entretiens (publié pour la première fois en 1787) entreprend de fonder la connaissance des lois de la nature sur la connaissance de l'essence de Dieu. L'interprétation des thèses de Spinoza dans l'Éthique et leur confrontation avec celles de Leibniz, de Jacobi, de Lessing, permet à Herder de présenter sa conception de Dieu. L'argument de l'ouvrage (un défenseur de Spinoza s'entretient avec un de ses détracteurs qu'il ne tarde pas à convaincre) montre que cette conception trouve son fondement dans la pensée de Spinoza, sans toutefois s'y réduire. L'interprétation avancée par Herder est pour une part critique. Il s'agit davantage pour lui, déclare-t-il, de retrouver l'esprit de Spinoza, que de s'attarder à la lettre de ses thèses, parfois obscurcie, en partie par une langue trop rigide (premier entretien) et en partie par sa situation historique. Herder s'oppose ainsi à la réduction de la matière à l'étendue (deuxième entretien) : ce serait là simplement un emprunt au vocabulaire cartésien, dont Spinoza n'aurait pas réussi à se défaire, alors même que sa pensée allait dans un tout autre sens, que Herder s'emploie à dévoiler. La critique porte en fait sur un point essentiel : si la matière est comprise comme étendue, la compréhension de l'essence divine ne permet pas, à elle seule, de penser la nature. Le dualisme entre pensée et étendue resterait, selon Herder, irréductible. Il s'agit donc de trouver un principe qui, essentiel à la nature divine, régisse l'ensemble de la Création. Seul un tel principe permet en effet de résoudre le difficile problème du rapport de Dieu et de sa création (dont l'exposé fait l'objet des troisième et quatrième entretiens). Il est découvert dans la force, force substantielle ou force organique. Dieu est ainsi pensé comme force s'exprimant dans sa Création par une infinité de forces. La nature révèle donc Dieu dans chacune de ses manifestations. Ce même point permet en outre de réfuter l'harmonie préétablie de Leibniz. Celui-ci n'a pas, selon Herder, tiré les conclusions qui s'imposaient d'après son propre système, où la matière aurait été "déjà animée de forces immatérielles " (p. 75). Le monde doit donc être conçu comme un système de forces qui interagissent, et dont toutes expriment la divinité comme force.

Ce principe unique étant acquis, Herder s'emploie à déterminer la nature de la nécessité qui le régit. Il écarte à cette fin plusieurs conceptions de Dieu. Dieu ne saurait être, comme l'affirme Moses Mendelssohn, " une dénomination collective de toutes les forces de l'entendement et de la pensée » (p. 86). Il ne serait alors qu'un simple nom, or l'existence est le concept qui permet de penser Dieu (quatrième entretien). Mais Dieu n'existe pas pour autant hors de sa création. Herder critique ainsi la conception jugée anthropomorphique d'un Dieu préexistant à son œuvre et la contemplant après l'avoir créée. Il exclut par là la pensée d'un Dieu créant d'après un principe du meilleur qui lui serait extérieur: le meilleur et la nature divine, en effet, ne font qu'un. Une telle réfutation présente l'intérêt de déterminer la voie à suivre par la science de la nature. Si Dieu n'a pas créé en choisissant entre différents possibles, il est vain de chercher à connaître la nature en s'interrogeant sur les intentions divines. Il faut plutôt étudier la nature des choses, d'après les lois qui la régissent. L'exigence scientifique est ainsi fondée dans la reconnaissance de la 
nécessité interne comme essence de la divinité : «Celui qui pourrait me montrer les lois naturelles et m'expliquer comment, par la nécessité interne et la conjonction des forces qui agissent en tels organes et non en tels autres, se forment nos phénomènes, ceux de la création vivante comme de celle que l'on dit morte, les sels, les plantes, les animaux et les hommes - celui-ci aurait bien plus contribué à la vénération, à l'amour et à l'admiration la plus magnifique qui soit de Dieu que celui qui me prêche, comme s'il le savait de la chambre haute de Dieu, que nous avons des pieds pour marcher, des yeux pour voir, et ainsi de suite " (p. 97). Une telle nécessité n'est pas une nécessité arbitraire, ou un fatalisme. Herder se réfère là encore à Spinoza pour établir que la nécessité divine est celle d'une pensée qui dépasse l'entendement humain. La liaison essentielle des choses, qui nous permet de les connaître, est celle d'une telle pensée (p. 117). Herder s'oppose ainsi à la conception d'une unification subjective développée par Kant. Dieu est donc le concept nécessaire, sans lequel il n'y a pas d'expérience possible. En retour, la nécessité dont on constate l'existence dans la nature vaut comme preuve de l'existence de Dieu.

Une fois cette conception de Dieu établie, Herder peut passer à l'élaboration des lois d'après lesquelles on connaît la nature. L'existence divine engendre l'existence de ses créatures, qui ne diffèrent entre elles que par la façon dont s'exerce la force divine. Chacune de ces créatures est ainsi parfaite en son genre. La nature doit être étudiée en terme de forces, lesquelles agissent organiquement. Herder donne ainsi un sens à l'expression de Lessing en kai pan (Un et Tout) : "Tout ce que nous appelons matière est donc plus ou moins en vie. C'est un règne de forces agissantes, qui forment un tout non seulement pour nos sens, en tant que phénomène, mais selon leur nature propre, et leur liaison. Dans ce tout, une seule force domine; autrement, il n'y aurait pas d'unité, pas de tout » (p. 136). Il faut donc, pour connaître la nature, déterminer la façon dont les forces agissent et s'organisent. Trois principes sont alors proposés : «1-Persévérance, c'est-à-dire persistance interne de tout être "; « 2-Union des semblables, et séparation des opposés »; «3-Assimilation à soi, et reproduction de soi en un autre " (p. 140). La connaissance métaphysique permet ainsi d'établir des principes de la philosophie de la nature, à partir de l'analyse de la divinité comme force, et de la force comme vie.

Ce concept de vie, peu développé, est cependant fondamental. Il n'est pas analysé, en effet, parce qu'il permet de penser l'unité réalisée entre ce qui peut sembler contraire. La citation, à l'intérieur ou à la fin de chaque entretien, de textes poétiques (Giulio C. Vanini, Heinrich von Kleist, Anthony Shaftesbury) sur Dieu ou la nature, illustrant l'idée centrale de l'ouvrage, manifeste ainsi sans doute la volonté de Herder de ne pas présenter des principes qui resteraient abstraits de l'expérience. La poésie sert par là de pierre de touche à l'élaboration d'une théorie qui prétend rendre compte de l'intuition du monde que chacun peut avoir (cinquième entretien).

Les solutions apportées par Herder peuvent être critiquées, en ce qu'elles permettent de résoudre les difficultés engendrées par les systèmes de Spinoza ou de Leibniz en procédant des assimilations qui posent à leur tour des problèmes. Il en est ainsi de l'emploi du terme de force, que relève dans sa présentation Myriam Bienenstock, comme concept à la fois physique et métaphysique (p. 8). Elles sont cependant d'une grande importance, non seulement par la défense à laquelle se livre Herder de la pensée de Spinoza contre ses nombreux détracteurs, mais surtout, par l'établissement de principes qui ont pu servir de guide à la Naturphilosophie. La 
Présentation de l'ouvrage le replace ainsi dans son contexte : la réponse aux Lettres sur la doctrine de Spinoza, de Jacobi manifeste l'évolution de la pensée de Herder et l'importance que cette pensée a prise pour ses contemporains, qu'ils la critiquent ou s'en inspirent.

Béatrice LENOIR

\section{HISTOIRE ET RELIGION}

Robin Cormack, Icônes et société à Byzance. Trad. de l'anglais par Marie-Odile Bernez. Brionne, Gérard Monfort, 1993. 17 × 24, 304 p., ill., bibliogr., index (Imago mundi).

Le présent livre est la traduction d'un ouvrage paru en 1985. Il ne prend pas la forme d'une écriture synthétique, mais d'une suite d'études centrées sur un personnage significatif d'un lieu et d'une époque. De cette façon, s'adressant à un vaste public, il tente de faire revivre concrètement la société byzantine à travers les documents qui sont le mieux conservés, ceux issus de l'art religieux, puisque l'essentiel des archives de cet empire bien administré, mais entièrement disparu, ne nous est pas parvenu. Il part du postulat, à l'évidence exact, que, dans cette société dont une minorité de membres savait lire, le discours oral, par essence perdu, et les arts visuels, relativement bien conservés, jouaient un rôle primordial. Les images Robin Cormack définit au passage l'icône et élargit son propos à toutes les représentations - sont donc essentielles dans la sociêté byzantine, mais leur rôle social évolue dans le temps, ce qui justifie la démarche chronologique. Dans chaque chapitre, Cormack se montre soucieux de nous présenter les grandes évolutions politiques et institutionnelles qui marquent la société étudiée, pour que le lecteur puisse le suivre aisément.

Le premier chapitre, "Le saint visible: saint Théodore de Sykéôn ", nous entraîne dans la Galatie de la fin du vi ${ }^{e}$ et du début du viI ${ }^{e}$ siècle sur les traces d'un saint villageois et de la société qui l'entourait. Cormack commence par présenter le récit hagiographique qui nous fait connaître Théodore. Sa connaissance du dossier hagiographique est ici bien sommaire, car il fait de Théodore « l'un des plus grands (saints) de l'Église byzantine " (p. 22), alors qu'il ne figure pas dans le « top 365 » de la sainteté byzantine, le synaxaire du patriarcat, document qui présentait le ou les saints officiels du jour mentionnés à l'office. Il fait également de la vie écrite au début du vil' siècle par Georges, un disciple du saint, le seul document hagiographique, alors que nous disposons d'un dossier complet comprenant notamment le récit, écrit deux siècles plus tard, du transfert de la relique à Constantinople sur 